\title{
Hybridization Dynamics and Extensive Introgression in the Daphnia longispina Species Complex: New Insights from a High-Quality Daphnia galeata Reference Genome
}

\author{
Jana Nickel $\mathbb{D}^{1, *}$, Tilman Schell ${ }^{2}$, Tania Holtzem³ ${ }^{3}$ Anne Thielsch ${ }^{4}$, Stuart R. Dennis ${ }^{5}$, Birgit C. Schlick-Steiner ${ }^{3}$, \\ Florian M. Steiner ${ }^{3}$, Markus Möst ${ }^{3}$, Markus Pfenninger $\mathbb{D}^{2,6,7}$, Klaus Schwenk ${ }^{4}$, and Mathilde Cordellier $\mathbb{D}^{1, *}$ \\ ${ }^{1}$ Institute of Zoology, Universität Hamburg, Germany \\ ${ }^{2}$ LOEWE Centre for Translational Biodiversity Genomics (LOEWE-TBG), Frankfurt am Main, Germany \\ ${ }^{3}$ Department of Ecology, University of Innsbruck, Austria \\ ${ }^{4}$ Molecular Ecology, Institute for Environmental Sciences, University Koblenz-Landau, Landau in der Pfalz, Germany \\ ${ }^{5}$ Department of Aquatic Ecology, EAWAG, Dübendorf, Switzerland \\ ${ }^{6}$ Molecular Ecology, Senckenberg Biodiversity and Climate Research Centre, Frankfurt, Germany \\ ${ }^{7}$ IoME, Gutenberg University, Mainz, Germany \\ *Corresponding authors: E-mails: jana.nickel@uni-hamburg.de; mathilde.cordellier@uni-hamburg.de.
}

Accepted: 24 November 2021

\begin{abstract}
Hybridization and introgression are recognized as an important source of variation that influence adaptive processes; both phenomena are frequent in the genus Daphnia, a keystone zooplankton taxon in freshwater ecosystems that comprises several species complexes. To investigate genome-wide consequences of introgression between species, we provide here the first high-quality genome assembly for a member of the Daphnia longispina species complex, Daphnia galeata. We further resequenced 49 whole genomes of three species of the complex and their interspecific hybrids both from genotypes sampled in the water column and from single resting eggs extracted from sediment cores. Populations from habitats with diverse ecological conditions offered an opportunity to study the dynamics of hybridization linked to ecological changes and revealed a high prevalence of hybrids. Using phylogenetic and population genomic approaches, we provide first insights into the intra- and interspecific genome-wide variability in this species complex and identify regions of high divergence. Finally, we assess the length of ancestry tracts in hybrids to characterize introgression patterns across the genome. Our analyses uncover a complex history of hybridization and introgression reflecting multiple generations of hybridization and backcrossing in the Daphnia longispina species complex. Overall, this study and the new resources presented here pave the way for a better understanding of ancient and contemporary gene flow in the species complex and facilitate future studies on resting egg banks accumulating in lake sediment.
\end{abstract}

Key words: introgression, hybridization, resting eggs, species complex, whole-genome amplification, genome assembly.

\section{Introduction}

Gene flow between species can be pervasive and can affect substantial parts of the genome. Hybridization and introgression are recognized as an important source of variation that can influence adaptive processes in plants, animals, yeast, and fungi (reviewed in Abbott et al. [2013]; Arnold and Martin [2009]). The amount of realized gene flow varies among taxa and along the genome; it is governed by intrinsic genomic features such as recombination rate, structural variation, and intrinsic incompatibilities, as well as the species' biology and ecology including ecological and sexual selection, migration, and mode of reproduction.

How can species in diversifying clades frequently hybridize and show introgression but nevertheless maintain species boundaries? A growing body of literature provides examples for a high variety of systems where speciation occurs in the

(C) The Author(s) 2021. Published by Oxford University Press on behalf of the Society for Molecular Biology and Evolution.

This is an Open Access article distributed under the terms of the Creative Commons Attribution License (https://creativecommons.org/icenses/by/4.0/), which permits unrestricted reuse, distribution, and reproduction in any medium, provided the original work is properly cited. 


\section{Significance}

Hybridization and introgression frequently occur in the water flea genus Daphnia. Until now, genomic resources to investigate the amount of introgression in the Daphnia longispina species complex, a widespread taxon in European lakes and keystone grazer of phytoplankton, were limited. We provide here a genome assembly and the first genomewide analysis of several species of the complex, based on samples from the water column and resting eggs from sediment cores. Using phylogenetic and population genomic approaches, we measure intra- and interspecific genomewide variability and identify regions of high divergence. Our study reveals extensive gene flow between taxa, and later generation hybrids originating from several rounds of backcrossing. It paves the way for further studies aiming at understanding how species boundaries are maintained in the face of gene flow.

face of gene flow (Fraïsse et al. 2014; Meier et al. 2017; Martin et al. 2019). However, it is important to recognize that these systems are distributed along a wide spectrum. On one side of this spectrum, hybridization occurs but is not followed by introgression for several reasons such as reduced hybrid fertility or strong selection against hybrid phenotypes, leading to rapid hybrid breakdown. Barth et al. (2020) found that species boundaries in tropical eels are stable despite millions of years of hybridization, and also observed very few admixed individuals beyond F1 and first-generation backcrosses. The hybrid breakdown observed in this system reduces the likelihood of introgression via backcrossing. On the other side of the spectrum, hybridization is followed by introgression, and ongoing exchange of genetic information between species (Martin et al. 2013; Butlin et al. 2014; Doellman et al. 2018; Kaiser et al. 2021). Several empirical studies (Canestrelli et al. 2017; Schreiber and Pfenninger 2021) as well as theoretical models (Yeaman and Whitlock 2011; Flaxman et al. 2014; Rafajlović et al. 2016) suggest the possibility of intermediate constant equilibrium states, meaning that certain parts of the genome remain diverged (islands or continents of divergence), whereas others are freely exchanged among closely related species without ever reaching complete genomic isolation.

Recurrent hybridization and introgression are frequent in the genus Daphnia (Crustacea, Cladocera) Members of the genus have served as ecological model organisms for over a century (Miner et al. 2012), and the first crustacean genome to be sequenced was that of a member of the Daphnia pulex species complex (Colbourne et al. 2011). Since then, the genomes of 45 crustaceans have been sequenced with a focus on species of economic or medical interest (NCBI, last accessed January 2021). Despite their key role in marine and freshwater food webs around the globe, genomic resources for zooplanktonic species are still scarce. In many aquatic food webs, zooplanktonic crustaceans link primary production by phytoplankton and secondary consumers, such as planktivorous fish and larger invertebrate species (Gannon and Stemberger 1978; Gliwicz 1990; Lampert and Sommer 2007).
Daphnia are highly phenotypically plastic and a textbook example for inducible defense mechanisms (Tollrian and Harvell 1999), as they respond to variation in predation risk through spectacular changes in morphology. Further, Daphnia are cyclical parthenogens and hence able to alternate between asexual and sexual reproduction. They reproduce asexually through longer periods of time, and the product of sexual reproduction events (usually seasonal) are resting eggs able to withstand adverse conditions for decades and even centuries (Frisch et al. 2014). Resting eggs extracted from sediment cores can be hatched, and ancient genotypes brought to life (reviewed in Orsini et al. 2013]). Moreover, the DNA preserved in those resting eggs can be directly analyzed with various molecular methods (Cousyn et al. 2001; Lack et al. 2018; Dziuba et al. 2020). Thus, cyclical parthenogenesis, biological archives in lake sediments and high levels of phenotypic plasticity make Daphnia a particularly interesting model for evolutionary studies.

The genus Daphnia is composed of two subgenera, Ctenodaphnia and Daphnia, and two groups are delimited within the subgenus Daphnia: the D. pulex group sensu lato and the Daphnia longispina group sensu lato (see Adamowicz et al. [2009]). The latter is sometimes also referred to as subgenus Hyalodaphnia and includes the $D$. longispina species complex (DLSC) (Petrusek, Hobæk, et al. 2008). The two Daphnia groups are highly differentiated and share their most recent common ancestor around $30 \mathrm{Ma}$ (MRCA $D$. longispina-D. pulex group, MRCA D. longispina-D. pulex group, Cornetti et al. 2019). Members of the genus Daphnia show little variation in chromosome number, with most species having ten pairs of chromosomes, except for the $D$. pulex group with $n=12$ (Trentini 1980; Beaton and Hebert 2004). All sequenced and assembled Daphnia genomes so far belong either to the D. pulex group or the subgenus Ctenodaphnia, however no high-quality reference genome of the third major group, the D. longispina group (Hyalodaphnia) is published.

The prevalence of hybridization in the genus Daphnia across taxa and ecosystems and its impact on their evolutionary history has intrigued researchers for decades (Wolf 1987; Schwenk 1993; Vergilino et al. 2011). In contrast to many 
other well-studied hybrid systems (Barton and Hewitt 1985) with clear defined hybrid zones where species' ranges overlap, the distribution of Daphnia species and their hybrids is more of a fragmented nature: they occupy lake and pond ecosystems that vary in their ecological characteristics and hence constitute a mosaic across the landscape. Ecologically differentiated taxa and their hybrids are thus distributed across habitat patches (Harrison 1986). Within these patches, the possibility to interrogate biological archives also revealed fluctuations in Daphnia community composition over time (Brede et al. 2009; Alric et al. 2016), associated with hybridization events among species in some cases. Variation in hybridization events across time and among habitats has often been observed in correlation with ecological changes, such as eutrophication or global change (Keller et al. 2008; Brede et al. 2009; Rellstab et al. 2011; Spaak et al. 2012; Dziuba et al. 2020; Cordellier et al. 2021).

Members of the DLSC inhabit many large ponds and lakes in central and northern Europe, and three of them have been particularly well studied: Daphnia galeata, D. longispina, and Daphnia cucullata (Petrusek, Hobæk, et al. 2008). These species can coexist, but earlier studies suggest gene flow among them is limited (Spaak 2004). Despite their obviously ancient divergence (Schwenk et al. 2000), DLSC species are still able to form interspecific hybrids, although not all combinations are equally likely to lead to viable and fertile individuals (Schwenk et al. 2001). A mechanism preventing gene flow among species might be their different ecological preferences, for example, regarding trophic level (Spaak et al. 2012), food quality (Seidendorf et al. 2007), and predation pressure (Spaak and Hoekstra 1997; Petrusek, Seda, et al. 2008).

Up to now, genetic markers available to study hybridization in the DLSC are limited to allozymes (Wolf and Mort 1986), a few mitochondrial regions (Schwenk 1993), a dozen microsatellite markers (Brede et al. 2006; Thielsch et al. 2012) and a few further nuclear loci (Billiones et al. 2004; Skage et al. 2007; Rusek et al. 2015). Seminal studies such as Brede et al. (2009) and Limburg and Weider (2002) first made use of microsatellite markers to analyze environmentally driven shifts in allelic frequencies, species, and hybrid composition of the DLSC communities in Lake Constance and Belauer See over time, respectively. Further, a number of studies addressed the spatial distribution of DLSC species/taxa with these markers (Griebel et al. 2016; Thielsch et al. 2017; Ma et al. 2019). These low-resolution markers allowed to identify hybrid individuals and brought evidence for introgression but could not provide the resolution necessary to either assess how pervasive introgression is or how it varies across the genome. Further, it is not clear whether introgression occurs among all three species to the same extent. Given the ubiquitous hybridization among the DLSC taxa, the question also arises why they are still well distinguishable species. Whether the DLSC represents a case of incipient speciation, introgression after secondary contact, speciation reversal, or has reached an intermediate constant equilibrium state, among other possibilities, can only be answered with genome-wide analyses empowered by a high-quality genome assembly.

Here, we present a high-quality assembly for $D$. galeata, thus filling an important gap for Daphnia whole-genome studies. Furthermore, to facilitate genome-wide assessments of divergence across species and of introgression between species, we conducted genome-wide resequencing studies in the DLSC. We analyzed whole-genome sequences of parental species and their interspecific hybrids, both from genotypes obtained in the wild and maintained in laboratories, and from single resting eggs extracted from sediment cores. We provide first insights into the intra- and interspecific genome-wide variability in this species complex and identify regions of high divergence. We reconstructed the phylogenetic relationships in the species complex using whole mitochondrial genomes. Finally, we assess the length of ancestry tracts in different classes of hybrids to characterize introgression patterns. Our study paves the way for long-awaited analyses on the dynamics of introgression in this complex and exploitation of the unique opportunity this group has to offer: a window of more than one hundred years of evolution in action.

\section{Results}

\section{Genome Assembly}

The raw assembly was obtained by combining PacBio long reads $(1,679,290,11.52 \mathrm{~Gb})$ and Illumina short reads (70,310,338, $9.79 \mathrm{~Gb}$ after trimming) and using the hybrid assembler RA (https://github.com/lbcb-sci/ra, last accessed January 2021). It originally comprised 1,415 contig sequences covering a total length of 153.6 Megabases (Mb), with an N50 value of 172 kilobases (kb) and a slightly elevated GC content $(40.02 \%$ supplementary table S3, Supplementary Material online) compared with the values expected for a Daphnia species (see table 1). According to an analysis based on coverage and GC content of the contig sequences conducted with blobtools (Laetsch and Blaxter 2017), a portion of the assembly consisted of non-Daphnia contigs, which could then be removed (267 contigs, equaling $22.97 \mathrm{Mb}$ ). Consequently, GC content decreased to $38.75 \%$, nearing the values obtained for other Daphnia assemblies (see table 1 for an overview). The application of this filter as well as the exclusion of the mitochondrial genome led to a decrease in the number of sequences and the total length of the assembly. Iterative scaffolding led to a decrease in the total number of sequences. This together with a substantial increase in N50 resulted in a highly contiguous assembly, with a total length of 133,304,63 bp, an N50 of $756.7 \mathrm{~kb}$ and only 346 sequences, that is, on an average 30 sequences per chromosome. Contiguity statistics for the different assembling steps are 
Table 1

Assembly Metrics and Annotation Statistics for the Present Assembly and Two Previously Published Daphnia Assemblies

\begin{tabular}{|c|c|c|c|}
\hline Species & D. galeata & D. pulex (Ye et al. 2017) & D. magna (Lee et al. 2019) \\
\hline Strain & M5 & PA42 & SK \\
\hline \multicolumn{4}{|l|}{ Assembly metrics } \\
\hline No. scaffolds & 346 & 493 & 4,192 \\
\hline Largest scaffold (bp) & $2,950,711$ & $7,584,612$ & $16,359,456$ \\
\hline Total length (bp) & $133,304,630$ & $189,550,516$ & $122,937,721$ \\
\hline N50 (bp) & 756,671 & $1,160,003$ & $10,124,675$ \\
\hline L50 (bp) & 48 & 36 & 5 \\
\hline GC (\%) & 38.75 & 40.39 & 40.54 \\
\hline No. N's & 120,845 & $4,006,006$ & $82,97,703$ \\
\hline No. N's per 100 kb & 90.65 & $2,113.42$ & $6,749.52$ \\
\hline \multicolumn{4}{|l|}{ Annotation } \\
\hline \multicolumn{4}{|l|}{ Number } \\
\hline Gene & 15,845 & 18,440 & 15,721 \\
\hline mRNA & 16,774 & 18,440 & 15,721 \\
\hline Exon & 117,364 & 128,688 & 95,203 \\
\hline CDS & 119,402 & 118,916 & 94,047 \\
\hline \multicolumn{4}{|l|}{ Mean } \\
\hline mRNAs/gene & 1.06 & 1 & 1 \\
\hline Exons/mRNA & 7.00 & 6.98 & 6.06 \\
\hline CDSs/mRNA & 7.12 & 6.45 & 5.98 \\
\hline \multicolumn{4}{|l|}{ Median length (bp) } \\
\hline Gene & 2,097 & $1,919.5$ & 1,586 \\
\hline mRNA & 2,142 & $1,919.5$ & 1,521 \\
\hline Exon & 167 & 162 & 160 \\
\hline Intron & 74 & & \\
\hline CDS & 152 & 144 & 159 \\
\hline \multicolumn{4}{|l|}{ Total space (bp) } \\
\hline Gene & $51,689,473$ & $53,936,938$ & $37,505,261$ \\
\hline mRNA & $51,689,329$ & $53,936,938$ & $36,178,687$ \\
\hline Exon & $29,314,592$ & $30,208,483$ & $22,336,755$ \\
\hline CDS & $25,132,876$ & $23,586,918$ & $21,881,778$ \\
\hline \multicolumn{4}{|l|}{ Single } \\
\hline Exon mRNA & 663 & 144 & 1,775 \\
\hline CDS mRNA & 710 & 0 & 0 \\
\hline \multicolumn{4}{|l|}{ BUSCO $N=1,066$} \\
\hline $\mathrm{C}$ & 94.3 & 94.1 & 97.0 \\
\hline$S$ & 91.7 & 82.6 & 95.3 \\
\hline $\mathrm{D}$ & 2.6 & 11.5 & 1.7 \\
\hline $\mathrm{F}$ & 0.7 & 3.5 & 1.7 \\
\hline$M$ & 5.0 & 2.4 & 1.3 \\
\hline DOGMA $N=4,222$ & 93.63 & 91.43 & 93.91 \\
\hline
\end{tabular}

NOTE-Contiguity statistics of the annotation were calculated excluding tRNAscan results. BUSCO 3.0 .2 was executed in protein mode for the different MAKER rounds. Conserved domain arrangements were searched with Pfam scan 1.6 and DOGMA 3.4. Results for BUSCO and DOGMA completeness statistics are given in percent.

given in supplementary table S3, Supplementary Material online.

Mapping the filtered Illumina reads with bwa mem (Li 2013) and PacBio reads with Minimap 2.17 (Li 2018), resulted in a mapping rate of, respectively, $94.1 \%$ and $85.5 \%$. The coverage distribution can be seen in supplementary figure $\mathrm{S} 1 B$, Supplementary Material online.

According to blobtools results, no contamination could be identified in the final assembly (supplementary fig. S1A,
Supplementary Material online). Remaining scaffolds (12, amounting to a total length $1.79 \mathrm{Mb}$ ) with taxonomic assignment other than Arthropoda were kept because coverage and GC are similar to $D$. galeata scaffolds and taxonomic assignment alone might be false positive. Further, the completeness assessment through BUSCO (Simão et al. 2015, Arthropoda set, odb9) indicated $95.7 \%$ of complete single copy core orthologs and a very low duplication rate (C: $95.7 \%$ [S: $94.7 \%, \mathrm{D}: 1.0 \%], \mathrm{F}: 0.8 \%, \mathrm{M}: 3.5 \%, \mathrm{n}: 1,066)$. The 
genome size was estimated based on mapped nucleotides and mode of the coverage distribution by backmap 0.3 (https://github.com/schellt/backmap, last accessed January 2021), resulting in 156.86 and $178.03 \mathrm{Mb}$ for Illumina $(52 x)$ and PacBio $(26 x)$ respectively, and by k-mer based approach using GenomeScope resulting in a size of $150.6 \mathrm{Mb}$.

When compared with other published full genomes for Daphnia species, the $D$. galeata final assembly is shorter than both D. pulex assemblies (Colbourne et al. 2011; Ye et al. 2017), and roughly the same size as Daphnia magna (Lee et al. 2019), which also has ten chromosomes (table 1). The GC content is lower, which can be attributed to the strict filtering for contamination applied pre- and postassembly, a procedure not applied in the other Daphnia assemblies, to our knowledge. Even though Lee et al. (2019) and Ye et al. (2017) treated the animals with antibotics before sequencing this suggests that these genome assemblies contain more contigs of bacterial origin than the $D$. galeata assembly. Thanks to the use of long-read data, iterative scaffolding and gap filling; the number and length of assembly gaps (Ns) are substantially lower and contiguity is high (but see supplementary table S3, Supplementary Material online).

\section{Genome Annotation}

After applying RepeatMasker (Smit et al. 2013-2015) with the custom repeat library described in the Materials and Methods section, $21.9 \%$ of the assembly was masked. The distribution of masked fraction per repeat element can be found in supplementary table S5, Supplementary Material online.

The final annotation with MAKER (Holt and Yandell 2011) predicts 15,845 genes with a median length of 2,097 base pairs. There is an average of 1.06 mRNAs per gene and seven exons per mRNA (table 1). The total number of predicted mRNA substantially differs from the number of transcripts previously published for this species $(32,903$, Huylmans et al. 2016). This is not surprising, as this transcriptome assembly did not make use of protein evidence we included here, and might contain isoforms. Further, it was based on a pool of mRNA from different clonal lines, and the assembly process might have been impeded by allelic diversity. As further quality criterion, the Annotation Editing Distance (AED) was compared across the three MAKER rounds and is visualized in supplementary figure S4, Supplementary Material online. AED improved mostly between rounds 1 and 2 of the annotation but only marginally with a further round.

A high percentage of protein sequences could be annotated: 15,898 (94.78\%) with InterProScan (Jones et al. 2014) and 15,960 (95.15\%) with BLAST against Swiss-Prot. With this combination of searches, a hit within InterProscan and BLAST was found for 16,675 protein sequences $(99.41 \%)$. GeneOntology annotation was possible for 9,555 sequences $(56.96 \%)$. A detailed overview of the functional annotated sequences per database or search algorithm is shown in supplementary table S7, Supplementary Material online.

\section{Genotyping}

Short-read sequence data were generated for 72 individuals: 17 unamplified DNA samples from isofemale clonal lines and 55 whole-genome amplification (WGA) samples (conducted on single resting eggs) that passed PCR contamination checks. After screening for contamination and removing data sets with only very few reads mapping to the $D$. galeata reference, 49 single genotypes remained: 32 from resting eggs and 17 from clonally propagated lines, established from individuals sampled in the water column or hatched from resting eggs (fig. $1 \mathrm{~A}$ and supplementary table $\mathrm{S} 2$, Supplementary Material online). Data gained from clonal lines with a species attribution were used as "parental species" data: five samples for $D$. galeata, four for $D$. longispina, and three for $D$. cucullata. The parental clones are part of two larger clone panels representing the parental species and their diversity in several European lakes. Their identity was established prior to this study either based on mitochondrial and microsatellite markers (M5, LC3_6, J2, Herrmann et al. 2017) or morphological examination, mitochondrial markers and factorial correspondence analyses based on microsatellite markers (Möst 2013; Alric et al. 2016), including hybrids and historical resting eggs, which separates parental species and hybrids (Dlouha et al. 2010; Rellstab et al. 2011; Yin et al. 2014; Alric et al. 2016). In addition, data were available for four resting eggs from Arendsee (AR), 12 resting eggs from Dobersdorfer See (DOB), five clonal lines and eight resting eggs from Eichbaumsee (EIC), and eight resting eggs from Selenter See (SE) (supplementary table S2, Supplementary Material online). Although the analysis of eggs from older sediment layers was attempted, biological material was either limited, of poor quality, or contaminated. Our isotope dating for DOB was unconclusive: either slides of the cored location or a high sedimentation rate meant the top $30 \mathrm{~cm}$ of the core did not show the usual isotope peaks, thus preventing precise dating. EIC samples were recent since they were collected from surface bank sand. For $\mathrm{SE}$, the oldest eggs analyzed here originated from the $2-3 \mathrm{~cm}$ layer of the core, which corresponds to max. $\sim 17$ years (Andersen T, personal communication). For $A R$ the oldest eggs for which results were obtained were isolated from the 4-5 cm layer, corresponding to 2005 (Balínt $M$, personal communication).

An average of $89.9 \%$ (range: $31.7-98.6 \%$ ) reads aligned to the reference genome with a mean coverage of $10.26 \times$ (range: 0.34-52.30x) (supplementary table S3, Supplementary Material online). The final SNP data set for subsequent analyses after quality-filtering included 3,240,339 SNPs across the 49 samples. To rule out possible reference bias, we compared mapping rates of reads with the reference allele and to the alternative allele at heterozygous sites. We found no 
A

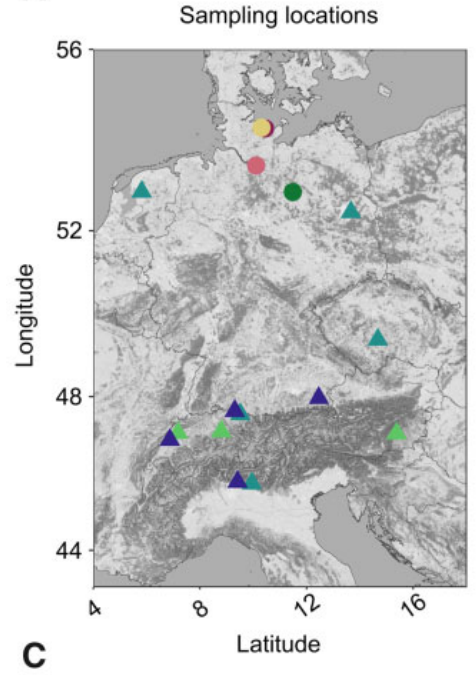

B

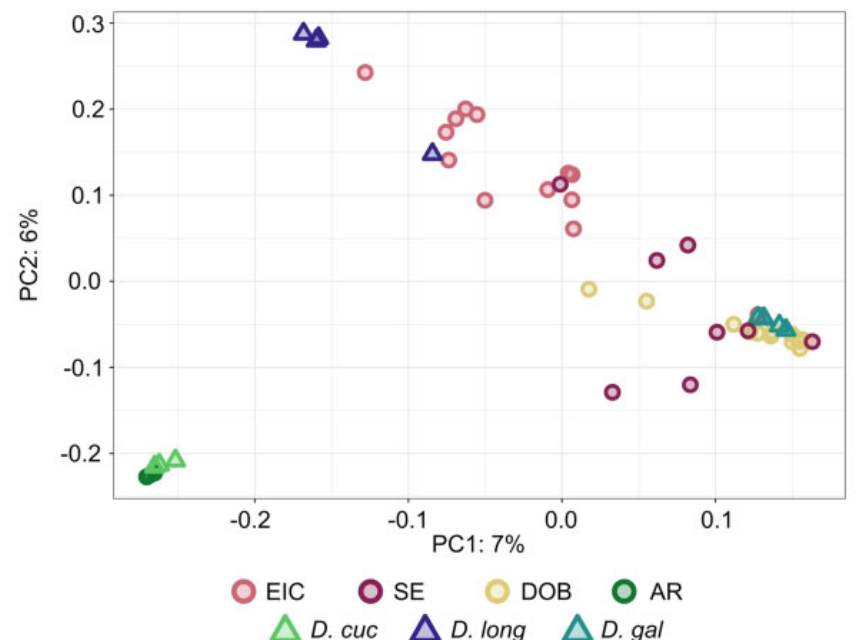

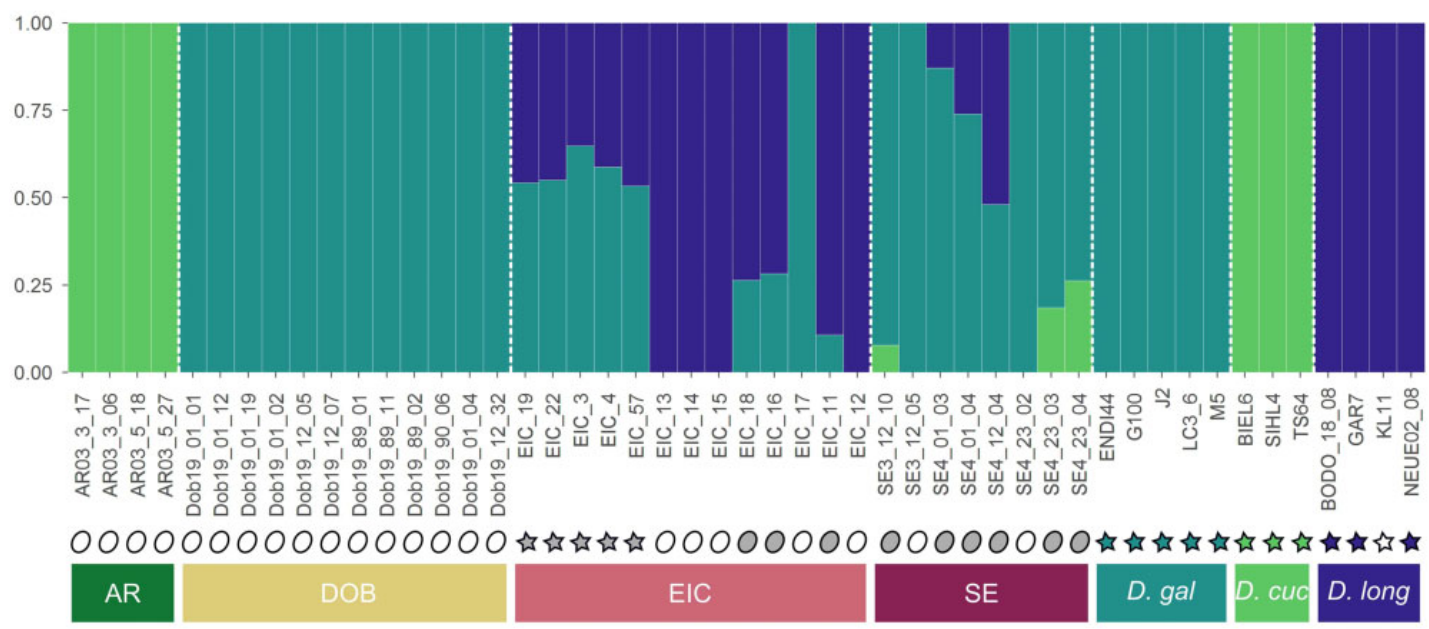

FIG. 1.-Parental species: Daphnia gal: D. galeata, D. long: D. longispina, D. cuc; D. cucullata, populations: AR, Arendsee; Dob, Dobersdorfer See; SE, Selenter See; EIC, Eichbaumsee. Color and symbol coding are consistent throughout panels $(A)$ and $(B)$. (A) Map of the sampling locations. (B) PCA plot obtained with SNPrelate, including loci with linkage $r^{2}<0.5$ within 500-kb sliding windows. (C) Admixture plot obtained with $K=3$. Symbols indicate the sample type: oval for genotypes sequenced directly from resting eggs, stars for genotypes sampled in the water column and propagated clonally in the laboratory prior to sequencing. Symbol filling indicates how these genotypes were classified in subsequent analyses: white for nonadmixed genotypes; grey for admixed genotypes; and green, blue, and teal for genotypes used as representatives for parental species. Bottom bars are color coded to match the color scheme used in panels $(A)$ and $(B)$.

preferential mapping of the reference allele, as all species categories and the hybrids had a median distribution close to 0.5 (supplementary fig. S2, Supplementary Material online).

\section{Principal Component Analysis}

In a PCA including all genotypes and conducted with SNPRelate v1.20.1 (Zheng et al. 2012), the parental species genotypes grouped in three very distinct clusters. Daphnia cucullata separated from $D$. galeata along PC1, which explained $7 \%$ of the variation. Daphnia longispina separated from $D$. galeata and $D$. cucullata along PC2 which explained $6 \%$ of the variation (fig. 1B). Although sampled in different lakes, all parental species genotypes were grouped in tight clusters along the two axes with little evidence for population substructure. Population AR clustered with the D. cucullata reference individuals while population samples from DOB, EIC, and SE were more spread out, mostly between the $D$. galeata and $D$. longispina clusters.

\section{Admixture Analyses Uncover Hybrids}

The PCA results are confirmed by an admixture analysis conducted with ADMIXTURE (Alexander and Lange 2011) with $K=3$, supported by the lowest cross-validation error of the tested $\mathrm{K}$ values. The known parental species genotypes were 
clearly separated into three clusters (fig. 1C). Although we detect no evidence of admixture in the AR and DOB samples and, based on our parental species, consider them to belong to the species $D$. cucullata and $D$. galeata, respectively, the two other populations seem to consist mostly of admixed individuals. The five EIC samples sequenced after clonal propagation were all found to be admixed ( $D$. galeata and $D$. longispina), whereas the EIC resting eggs were either admixed (3) or belonged to one of the parental species (5). SE resting eggs present all combinations of admixture except $D$. cucullata $\times D$. longispina: $D$. galeata (2), admixed between D. galeata and D. cucullata (3), and admixed between D. galeata and $D$. longispina (3).

\section{Ancestry Painting}

Based on results obtained in the ADMIXTURE analysis, two pairs of species and their putative hybrids were analyzed with an "ancestry painting" approach, outlined in Barth et al. (2020) and Runemark, Eroukhmanoff, et al. (2018): $D$. galeata and $D$. longispina parental genotypes and putative hybrids between them from populations EIC and SE, and $D$. galeata and $D$. cucullata parental genotypes and putative hybrids between them from population SE. Briefly, after identifying fixed sites for each of the species in the analyzed pair, heterozygosity was calculated for these sites and a hybrid index derived from the obtained results (https://github.com/ mmatschiner/tutorials/tree/master/analysis_of_introgression_ with_snp_data, last accessed November 2021). Further, information on the maternal species is used to tentatively categorize the admixed individuals. For a first-generation hybrid (F1) the expectation would be $50 \%$ of the nuclear genome being derived from each parental species (hybrid index $\approx 0.5$ ) and mostly heterozygous fixed sites (heterozygosity $\approx 1.0$ ). Individuals originating from the backcrossing of F1 with one of the parental species are expected to have hybrid index values around 0.25 or 0.75 (fig. 2D). We consider individuals with intermediate hybrid indices $(>0.25$ and $<0.75)$ and lower heterozygosity $(<0.5)$ to be later-generation hybrids, meaning they have one or multiple hybrid ancestors we are not able to classify further (Slager et al. 2020) We consider individuals with a hybrid index of $\leq 0.25$ or $\geq 0.75$ to be backcrossed with the respective parental species in at least one generation and the majority of the genome derives from one species. This definition is broad and will be refined with the addition of a greater number of parental genotypes.

The comparison of genotypes from parental species $D$. galeata and $D$. longispina (five and three individuals, respectively) allowed identifying a total of 335,052 fixed sites between the two species. Due to the quality filters applied to parental and hybrid genotypes, we could analyze 131,914 of these fixed sites in the putative hybrids, where read coverage was sufficient. The diploid genotypes were then plotted for all hybrids as homozygous for either of the parental species or as heterozygous (fig. 2A for the 50 longest scaffolds). The $D$. longispina reference clone KL11 was excluded from further analysis due to issues with missing data. All 11 genotypes from SE and EIC identified as likely $D$. galeata $\times D$. longispina hybrids in the ADMIXTURE analysis possessed a $D$. galeata mitochondrial genome. The proportion of the maternal $D$. galeata genome in these hybrids, however, varied greatly, between $27.4 \%$ and $86.6 \%$, and they all showed low heterozygosity, between $9.1 \%$ and $34.6 \%$ (fig. 2C and table 2). These values are unlikely for F1 hybrids or backcrosses of F1 with one of the parental species (table 2).

Comparing genotypes from the parental species $D$. galeata and $D$. cucullata (five and three individuals, respectively) led to identifying 715,438 fixed sites between the two species (due to quality filtering, 275,216 of these sites were further analyzed). All three $D$. galeata $\times D$. cucullata hybrids carried a $D$. cucullata mitochondrial genome, their hybrid index varied between 0.079 and 0.267 and their heterozygosity ranged from $6.4 \%$ to $50.9 \%$ (fig. $2 B$ and $C$; table 2). The individual SE_23_04 is most likely the result of a backcrossing with D. galeata; however, it is difficult to determine what backcrossed with it: either an F1 hybrid or a later generation hybrid, that is, that resulted from several generations of admixture. Haplotype information would be needed to gain certainty. The other two hybrids' lower hybrid index hints backcrossing with $D$. galeata, according to the criteria defined above.

\section{Population Genomics Parameters}

To calculate genome-wide nucleotide diversity $(\pi)$, betweentaxon differentiation $\left(F_{\mathrm{ST}}\right)$, and between-taxon divergence $\left(d_{\mathrm{xy}}\right)$ within $100-\mathrm{kb}$ sliding windows, we took advantage of the inference made with ADMIXTURE and pooled all genotypes which were unambiguously assigned to either of the parental species clusters. Consequently, a total of seven genotypes from four populations were classified as $D$. cucullata, eight from five populations as $D$. longispina, and 20 genotypes from eight populations as $D$. galeata (supplementary table $S 2$, Supplementary Material online). All values $\left(d_{x y}, \pi\right.$, and $F_{S T}$ ) were calculated with the script popgenWindows.py (github.com/simonhmartin/genomics_general, release 0.3, last accessed February 2021) for each species pair and are plotted for the 50 largest scaffolds in figure 3.

The window-based $F_{\mathrm{ST}}$ values for all three possible pairs among the three species averaged 0.274 for $D$. galeata versus $D$. longispina, 0.343 for $D$. cucullata versus $D$. longispina and 0.364 for $D$. galeata versus $D$. cucullata. The mean sequence divergence $d_{x y}$ for the three pairs was 0.018 for $D$. galeata versus $D$. longispina and 0.022 for both $D$. cucullata versus $D$. longispina and $D$. cucullata versus $D$. galeata. Both parameters show similar patterns, with lower values on an average when comparing $D$. galeata to $D$. longispina than when comparing cucullata to either one of the other species. These 
A

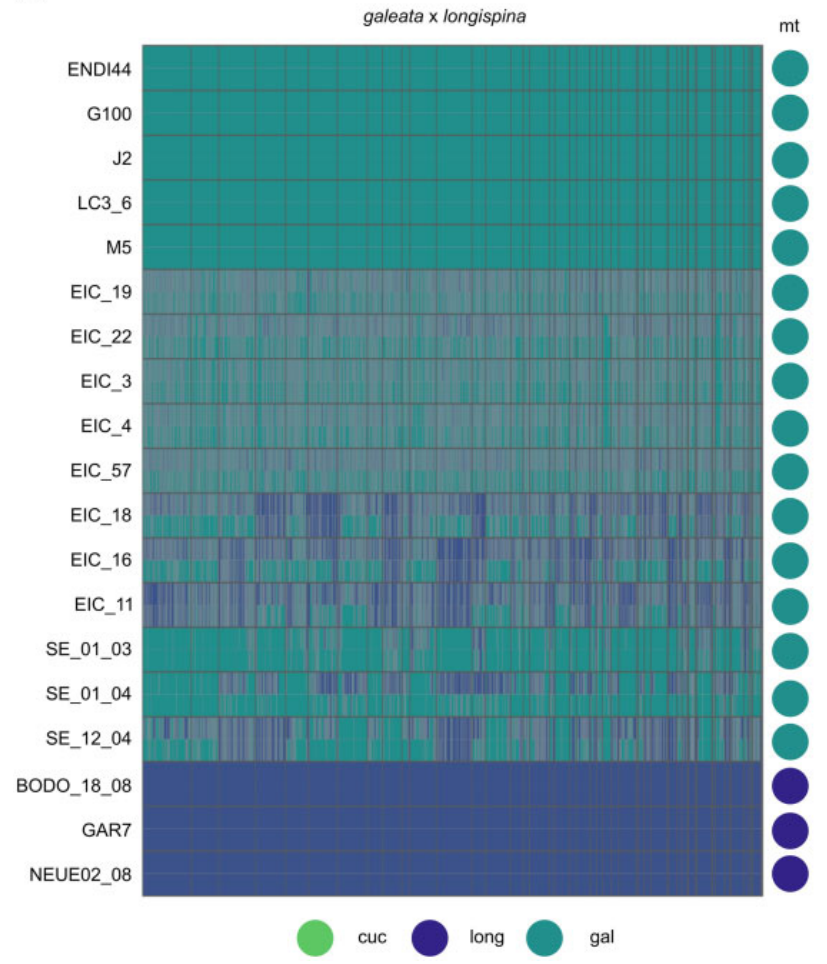

B
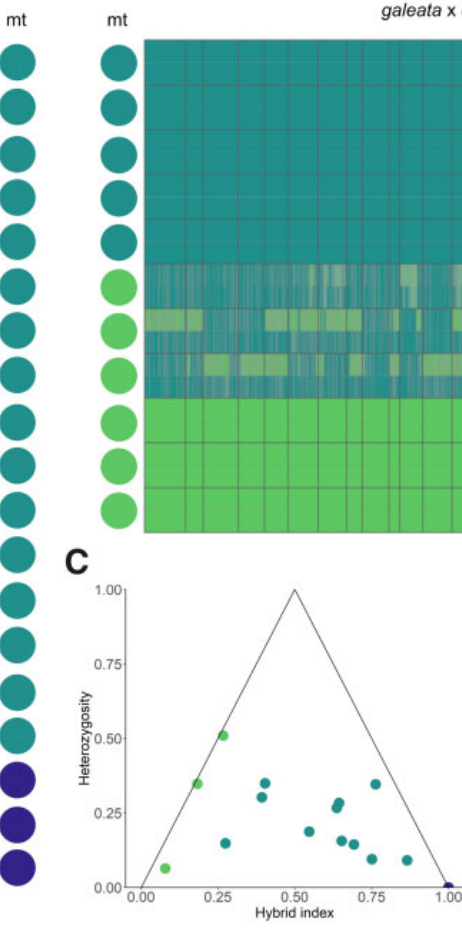

galeata $\times$ cucullata

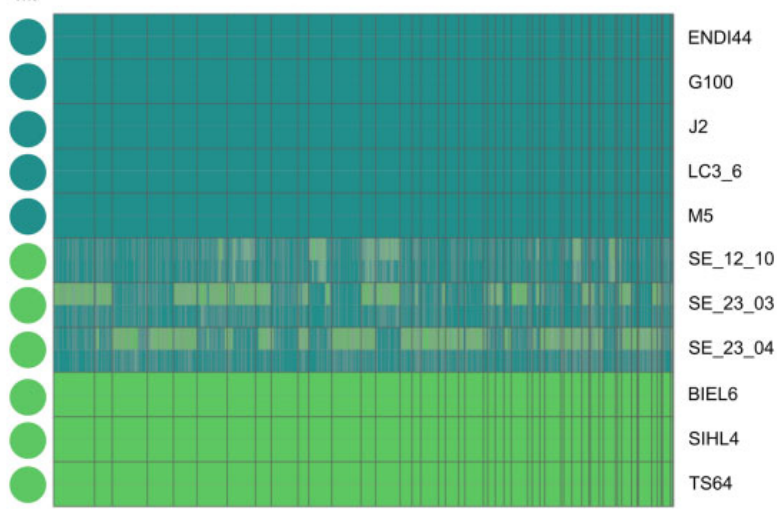

D

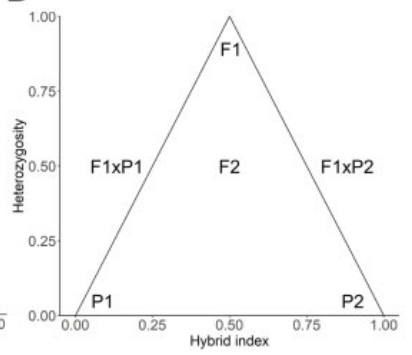

FIG. 2.- $(A, B)$ Ancestry painting of the hybrid individuals identified through the admixture analysis. Each row represents an individual. Colored circles on the side indicate the mitochondrial identity of the individuals, based on the analysis of full mitochondrial genomes. Scaffolds are sorted by length and separated by thin grey lines. In panels $(A)$ and $(B)$, the five upper rows represent individuals assigned to the parental species Daphnia galeata. In $(A)$, the last three rows correspond to individuals assigned to the parental species $D$. longispina. In $(B)$, the last three rows correspond to individuals assigned to the parental species $D$. cucullata. Triangle plots summarizing $(C)$ the hybrid index and mitochondrial species identity for all individuals identified as admixed, and (D) The hypothetical expected means of parental species (P1 and P2) and hybrid classes (F1xP1 and F1xP2: backcrosses with parental species P1 and P2, respectively).

Table 2

Data Derived from Ancestry Painting Analysis and Based on the Fixed Sites Inferred from Analyzing Parental Species Genotypes

\begin{tabular}{|c|c|c|c|c|c|}
\hline Sample & Hybrid Index & Heterozygosity & Maternal Species & Hybrid & Interpretation \\
\hline SE_12_10 & 0.079 & 0.064 & cuc & gal ×cuc & Backcross gal \\
\hline SE_23_03 & 0.183 & 0.348 & cuc & gal ×cuc & Backcross gal \\
\hline SE_23_04 & 0.267 & 0.509 & cuc & gal ×cuc & Unclear \\
\hline EIC_19 & 0.653 & 0.156 & gal & gal $\times$ long & Later-generation \\
\hline EIC_22 & 0.644 & 0.283 & gal & gal $\times$ long & Later-generation \\
\hline EIC_3 & 0.751 & 0.095 & gal & gal $\times$ long & Backcross gal \\
\hline EIC_4 & 0.693 & 0.144 & gal & gal $\times$ long & Later-generation \\
\hline EIC_57 & 0.637 & 0.267 & gal & gal $\times$ long & Later-generation \\
\hline EIC_18 & 0.393 & 0.302 & gal & gal $\times$ long & Later-generation \\
\hline EIC_16 & 0.403 & 0.349 & gal & gal $\times$ long & Later-generation \\
\hline EIC_11 & 0.274 & 0.148 & gal & gal $\times$ long & Later-generation \\
\hline SE_01_03 & 0.866 & 0.091 & gal & gal $\times$ long & Backcross gal \\
\hline SE_01_04 & 0.763 & 0.346 & gal & gal $\times$ long & Backcross gal \\
\hline SE_12_04 & 0.547 & 0.187 & gal & gal $\times$ long & Later-generation \\
\hline
\end{tabular}

Note.-Maternal species attribution is based on mitochondrial phylogeny, hybrid attribution is based on the ADMIXTURE plot.

patterns confirm the results obtained with other analyses, for example, the higher number of fixed sites between $D$. galeata and $D$. cucullata in the ancestry painting analysis.
The window-based estimates show high variability in levels of differentiation and divergence along the genome. Further, regions of high or low differentiation are mostly associated 


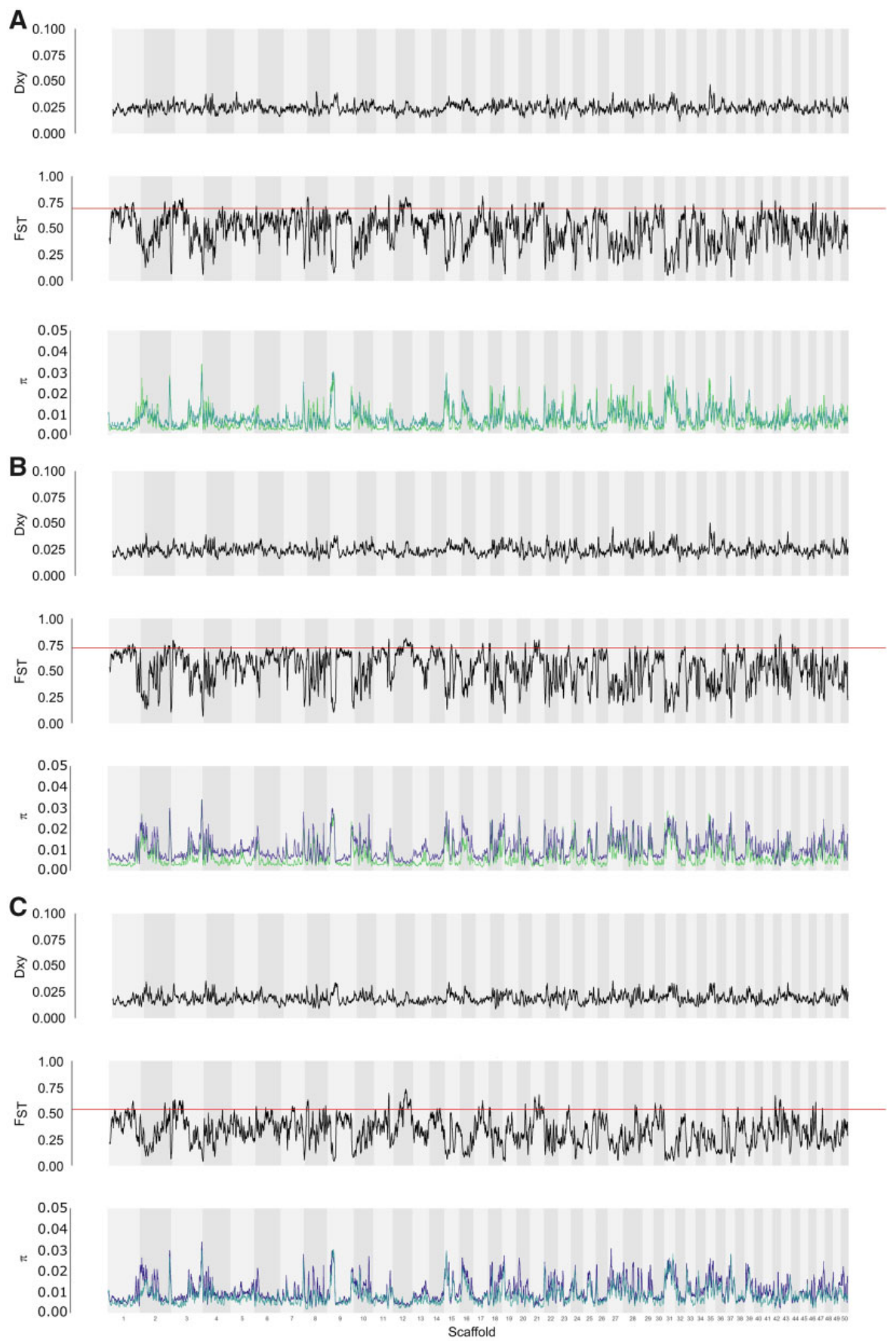

FIG. 3.-Window-based statistics for the pairs (A) Daphnia galeata/D. cucullata, (B) D cucullata/D. longispina, and (C) D. galeata/D. longispina, shown for the 50 largest scaffolds in 100-kb windows with 10-kb step size - calculations are for all nonadmixed individuals unambiguously assigned to parental species according to the ADMIXTURE analysis. In each panel from top to bottom: $d_{x y}$ values, pairwise $F_{\text {ST }}$ values with a red horizontal line indicating the 95 th percentile, nucleotide diversity $(\pi)$ for $D$. galeata (teal), D. longispina (dark blue), and D. cucullata (lime green).

with depleted or high nucleotide diversity, respectively (see scaffolds 2 and 9, for example). However, the genome being represented by unordered scaffolds instead of chromosomes makes this difficult to interpret further.

Nucleotide diversity $(\pi)$ to quantify the level of genetic variation within each taxon was on an average higher for
D. longispina $(1.18 \%)$ than for the other two species $(0.95 \%$ and $0.85 \%$ for $D$. galeata and $D$. cucullata, respectively). This cannot be explained by the differences in group sample sizes, since $D$. galeata was the group with the largest sample size (and highest number of sampled populations). To ensure our window-based estimates were not biased because 
of the overrepresentation of some populations in a group (e.g., DOB in the galeata group), we also calculated these indices using only one individual from each population per species; if one population contained multiple individuals, we picked one individual at random to represent this population (see supplementary table S2, Supplementary Material online, for a listing of the used genotypes-results shown in supplementary fig. S4, Supplementary Material online).

Many more highly differentiated windows and genes were shared among two or all species pairs than would be expected by a random intersection (supplementary fig. S5, Supplementary Material online). For example, a total of 2,575 10-kb windows had an $F_{\mathrm{ST}}$ value within the 95th percentile in the pair $D$. galeata/D. longispina and 2,569 in the pair $D$. galeata/D. cucullata. The mean expected number of windows in common between these two pairwise comparisons was 113, but the number of windows in common observed in the data was 1,601. A similar pattern was observed in all other intersections. This result suggests that the location of differentiated genome parts is not due to random processes but has biological significance. A GO-enrichment analysis of these isolated genes to shed light on the function of these species-specific genes, however, was not possible, because of the low number of genes with GO annotation. For the pair $D$. galeata/cucullata, only $12 \%$ of the genes in the outlier windows were annotated with Gene Ontologies, for the pair $D$. galeata/longispina it was $11 \%$ and for the $D$. cucullata/longispina pair it was $10 \%$.

\section{Phylogeny Based on Complete Mitochondrial Genomes}

Phylogenetic reconstruction based on the mitochondrial protein-coding and ribosomal RNA (rRNA) genes were largely consistent with earlier mitochondrial phylogenies based on single or few mitochondrial genes (Adamowicz et al. 2009; Petrusek et al. 2012). We identified highly supported clades comprising the respective parental genotypes, hence representing $D$. longispina, D. cucullata, and D. galeata mitochondrial haplotypes (fig. 4). Daphnia cucullata and D. galeata mitochondrial haplotypes clustered as sister groups. Although the mitochondrial haplotypes in the $D$. longispina and $D$. cucullata clusters do not show much divergence, the D. galeata haplotype cluster also contains deeper branching events (haplotype EIC_15 and AR3_17/AR5_18). Further, although all samples from AR were unequivocally categorized as D. cucullata in the ADMIXTURE analysis and clustered with $D$. cucullata parental genotypes in the PCA, two of them have mitochondrial haplotypes falling into the $D$. galeata cluster (AR3_17 and AR5_18). A similar mismatch was also observed for EIC_15, which clusters with $D$. longispina when considering nuclear SNP and with $D$. galeata when considering the mitochondrial genome. Within the species clusters, we observed a grouping by lake with many haplotypes being either identical or very similar when originating from the same location. The trees obtained with either only protein-coding genes (PCGs) (CODON model) or PCGs and rRNA genes but with a mixed model (DNA for rRNAs and CODON for DNA) were all consistent with the tree shown here and are therefore not included.

\section{Patterns of Introgression}

We tested all four northern Germany populations (EIC, DOB, $A R$, and SE) for admixture between the three reference species with $f_{3}$ statistics tests (table 3 ) and considered a $Z$ score $<-3$ as significant (following Reich et al. 2009; Patterson et al. 2012). Negative and significant values $\left(f_{3}=-0.19\right)$ using EIC as the test population and $D$. galeata and $D$. longispina as the source populations indicated mixed ancestry from these two or closely related populations. For population $\mathrm{SE}$, the $f_{3}$ test supports both admixed ancestry from $D$. galeata and $D$. longispina $\left(f_{3}=-0.09\right)$ and $D$. galeata and $D$. cucullata $\left(f_{3}=-0.15\right)$. All tests for population $D O B$ and $A R$ were positive providing no evidence of admixture events. The supported introgression events are consistent with the results in our previous analyses conducted with ADMIXTURE and the ancestry painting approach.

We performed local ancestry inference with Loter (DiasAlves et al. 2018) to trace genome-wide introgression among the hybrids and infer additional details about the parental species and backcross history from haplotype information. The results were summarized genome-wide for the ancestry proportion, heterozygosity of ancestry and the number of ancestry transitions where each ancestry tract is counted when the state of an SNP changes to the other species or at the end of a scaffold (fig. 5). The three $D$. galeataxcucullata hybrids were all found to have high galeata ancestry (73.994.2\%) and the individuals SE_23_03 and SE_23_04 were confirmed as the offspring of a later-generation hybrid and a pure galeata parent (supplementary fig. S6B, Supplementary Material online).

Seven $D$. galeata $\times$ longispina hybrids had very high galeata ancestry (81.4-97.9\%), and visual inspection of the ancestry tracts (supplementary fig. S6A, Supplementary Material online) revealed very short longispina tracts and scaffolds with multiple breakpoints indicating multiple generations of recombination. Four D. galeata $\times$ longispina hybrids had lower galeata ancestry (27.7-59.0\%) and the presence of complete longispina scaffolds implying some backcrossing with longispina. The haplotype phasing confirmed that the parents of all $D$. galeata $\times$ longispina hybrids were also of hybrid origin. The average and maximum ancestry tract length for all D. galeata $\times$ longispina hybrids is shorter than those for D. galeata $\times$ cucullata hybrids. 


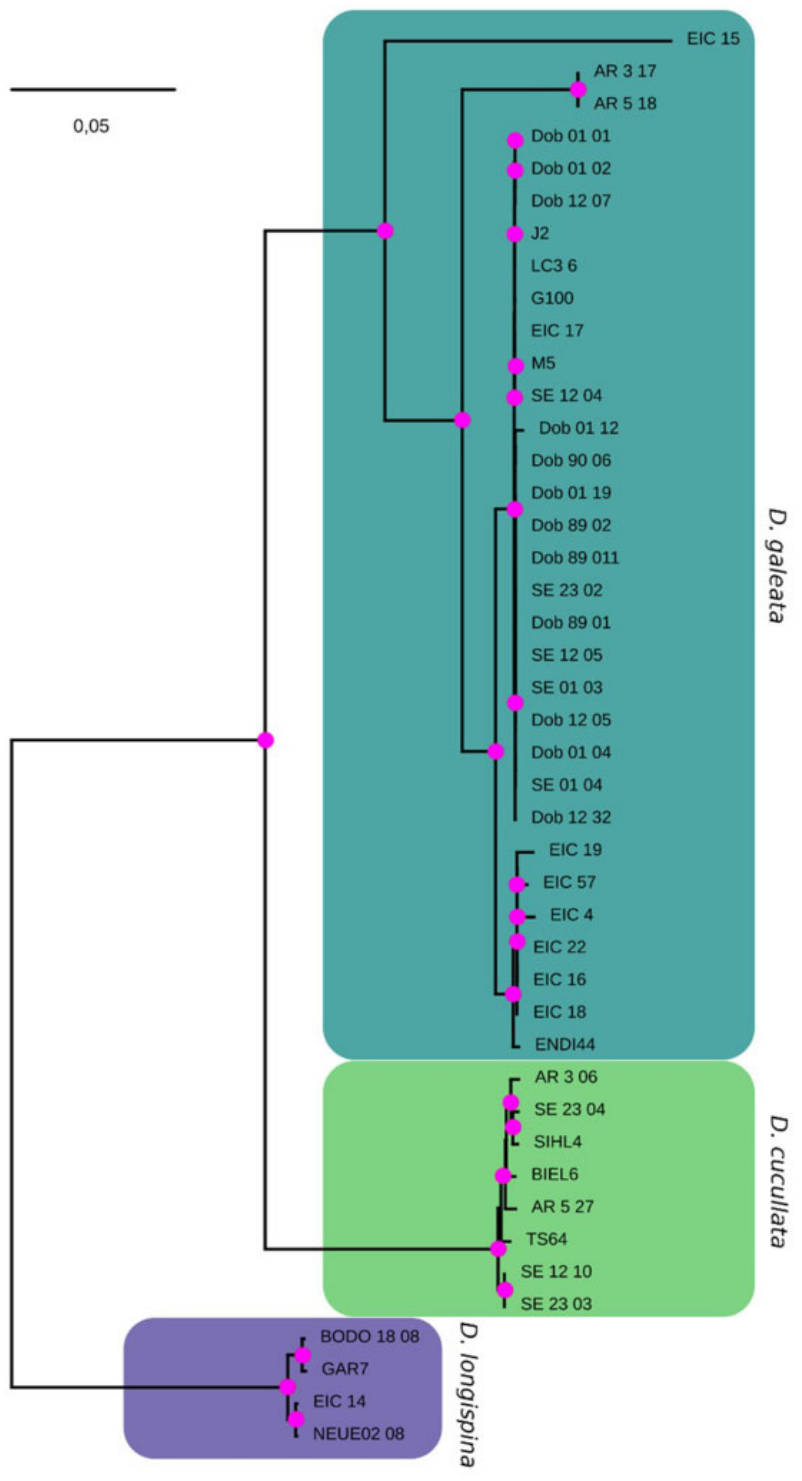

FIG. 4.-Maximum-likelihood tree reconstructed from mitochondrial PCGs and rRNA genes of parental species, clones sampled in the water column and resting eggs sequenced in this study. The tree reveals distinct and highly supported clusters corresponding to Daphnia galeata, $D$. cucullata, and $D$. longispina mitotypes (as defined by the respective parental species and a sister taxa relationship between $D$. galeata and $D$. cucullata). Here, the best tree ( $\log L=-47,950.82$ ) rooted with outgroup $D$. laevis is depicted. Magenta dots indicate Shimodaira-Hasegawa approximate likelihood ratio test values $\geq 80 \%$ and ultrafast bootstrap support values $\geq 95 \%$ calculated from 10,000 bootstrap replicates (SH-aLRT/ UFboot). The scale bar corresponds to 0.05 nucleotide substitutions per nucleotide site. KL11 was excluded due to missing data.

\section{Discussion}

\section{A Reference Genome for Studying a Species Complex}

Daphnia are a key species in freshwater habitats. Previous studies have established reference genomes for the model species D. pulex (Colbourne et al. 2011; Ye et al. 2017) and D. magna (Lee et al. 2019). No high-quality reference genome for species belonging to the DLSC was available so far. To date, it is unclear whether the ecological differentiation and/ or intrinsic incompatibilities drive and maintain divergence between DLSC species. Besides its utility for studies of hybridization events in the DLSC, the new assembly we present here will thus allow us to better understand the evolution of a key species in European freshwaters.

Even though the onset of the DLSC radiation was dated to 5-7 Ma based on nuclear and mitochondrial markers (Schwenk 1993; Taylor et al. 1996; Adamowicz et al. 2009), several factors confirm the suitability of this reference for all tested species. Mapping success and coverage of whole-genome data from $D$. cucullata and $D$. longispina to the reference genome were high, and we found no evidence of reference bias. This assembly clearly benefited from advances both in the sequencing technologies and assembly and postprocessing algorithms since the first Daphnia genome (Colbourne et al. 2011). The metrics used for assessing its quality reveal that in particular, the combination of longand short-read technologies led to highly contiguous and accurate scaffolds. Although we likely did not recover the genome in its full length (133 Mb out of an estimated $156 \mathrm{Mb}$ ), and the N50 value is lower than those obtained for $D$. pulex (Ye et al. 2017) and D. magna (Lee et al. 2019), iterative scaffolding allowed for a very efficient gap-closing, and an exceptionally low number of mismatches, compared with the other Daphnia assemblies.

\section{Pervasive Introgression in the DLSC}

We utilize a method that allows us to interrogate biological archives and analyze whole Daphnia genomes directly from the resting egg bank (Lack et al. 2018) without hatching and culturing several clonal lineages. This provides a wide sweep of populations, past and present, with each egg being the product of local sexual recombination.

Although no evidence of introgression was found in the DOB population, the three other locations host a variety of admixed genotypes. SE \& EIC can even be considered hybridization hotspots with more than $60 \%$ of individuals having hybrid ancestry, as revealed in the ADMIXTURE analysis. However, Kong and Kubatko (2021) very recently showed that ADMIXTURE is sensitive to unequal contributions by the parental species, and we thus sought to support these inferences by $f_{3}$ calculations and using an ancestry painting approach.

In DOB, ADMIXTURE delivered unequivocal results. Further, the $f_{3}$ index indicated that no introgression was detectable in this population. However, the PCA plot shows that some of the DOB genotypes are near hybrid individuals. ANGSD results are similar but these genotypes nearer the parental species (supplementary fig. S3A, Supplementary 
Table 3

Summary of the $f_{3}$ Statistic for Admixture in the Form (C; A, B)

\begin{tabular}{|c|c|c|c|c|c|c|}
\hline Source Population A & Source Population B & Target Population C & $f_{3}$ & SE & Z-Score & No. Sites \\
\hline gal & long & AR & 5.48226 & 0.154392 & 35.509 & $1,072,106$ \\
\hline gal & cuc & AR & 0.245124 & 0.006208 & 39.487 & 791,570 \\
\hline long & cuc & AR & 0.225393 & 0.005475 & 41.165 & 875,293 \\
\hline gal & long & Dob & 0.152725 & 0.003233 & 47.242 & 752,265 \\
\hline gal & cuc & Dob & 0.150027 & 0.003028 & 49.546 & 815,035 \\
\hline long & cuc & Dob & 1.763147 & 0.049657 & 35.506 & $1,059,418$ \\
\hline gal & long & $\mathrm{EIC}$ & -0.193114 & 0.003224 & -59.898 & 864,328 \\
\hline gal & cuc & EIC & 0.086614 & 0.002623 & 33.025 & $1,119,715$ \\
\hline long & cuc & EIC & 0.014322 & 0.001774 & 8.071 & $1,145,530$ \\
\hline gal & long & SE & -0.093233 & 0.003222 & -28.939 & $1,029,744$ \\
\hline gal & cuc & SE & -0.154696 & 0.003924 & -39.426 & 955,516 \\
\hline long & cuc & SE & 0.288212 & 0.011203 & 25.727 & $1,092,186$ \\
\hline
\end{tabular}

Note.-A significantly $\left(Z\right.$ score $<-3$, in bold) negative $f_{3}$ value implies that the target population $C$ is admixed. SE, standard error.

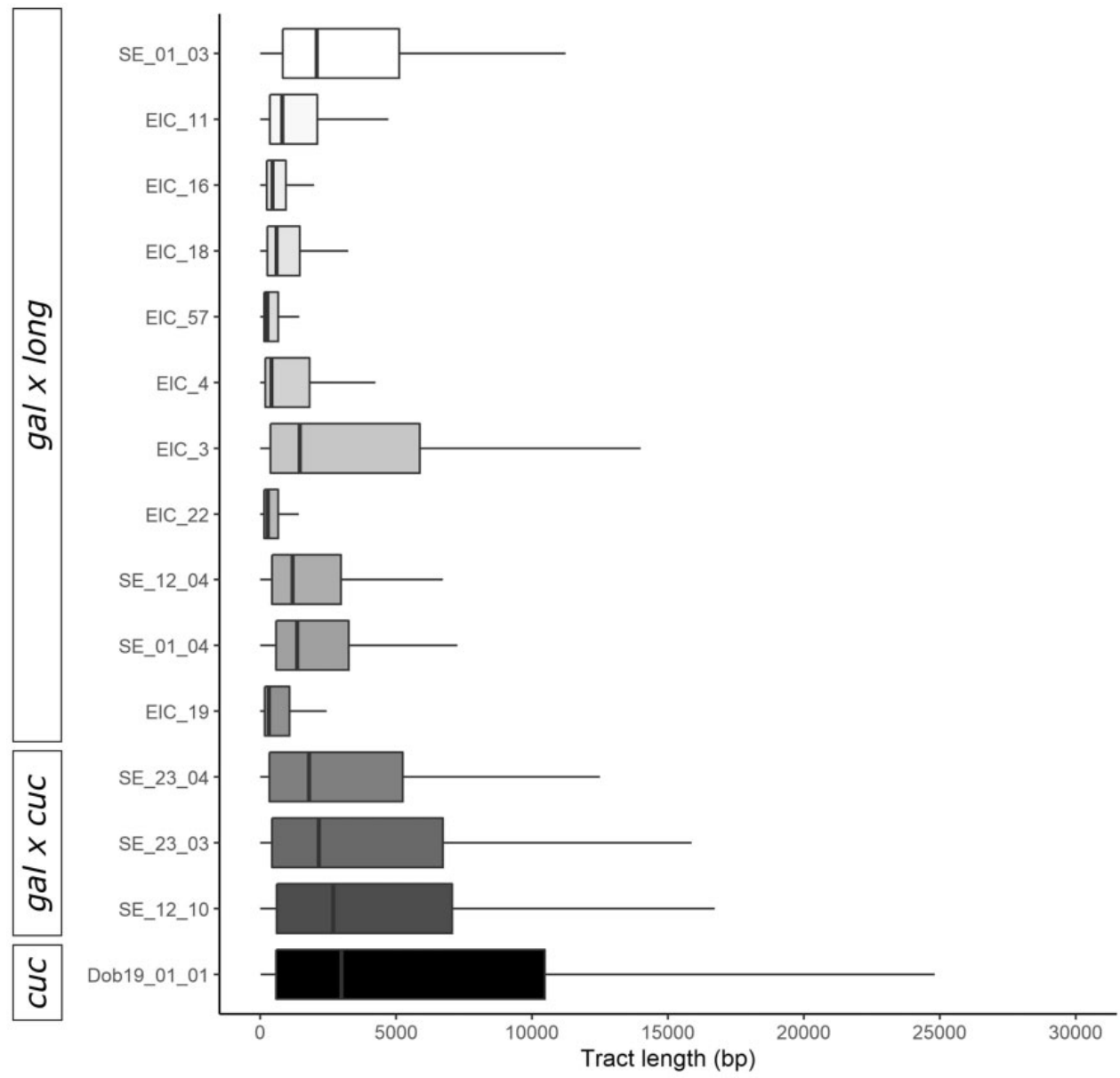

FIG. 5.-Distribution of the ancestry tract length where each ancestry tract represents the state of a SNP changing to the other species or the end of a scaffold in the local ancestry inference for each admixed individual and one nonadmixed $D$. cucullata individual. The nonadmixed individual displays the ancestry tract length distribution when all scaffolds derive from the same species. Hybrid type (according to ADMIXTURE analysis) is given on the left side.

Material online). To address these slightly contradictory results, we therefore conducted an ancestry painting on two DOB genotypes, (12_07 and 89_02, supplementary table
S7, Supplementary Material online). Both genotypes had a very low heterozygosity, thus confirming the ADMIXTURE and $f_{3}$ outcomes. A possible explanation would be that these 
two genotypes carry variation that is not reflected in our limited sampling of the parental species. When comparing with microsatellite-based analysis including many more populations and data points (Thielsch et al. 2009), the D. galeata cluster has often been larger and more diverse than the others. The seemingly two "stray" DOB genotypes are therefore likely well within the species variation boundaries. All mitochondrial haplotypes were clustered together in the phylogenetic reconstruction as well.

In $A R$, despite the high resting egg density found in the sediment, only very few could be successfully genotyped. Although all inferences based on nuclear markers (PCA, ADMIXTURE, $f_{3}$ ) indicated an absence of hybridization or introgression in this population, the mitochondrial phylogenetic reconstruction showed diverging results. From a nuclear point of view, all genotypes could be categorized as $D$. cucullata, but two out of four AR individuals presented the mitochondrial genome of another species, that is, D. galeata. However, the phylogenetic reconstruction shows that the two AR mitochondrial haplotypes form a cluster separate from the main D. galeata cluster, which hints at different evolutionary history for these mitochondrial genomes. Such distinct lineages within a species and mito-nuclear discordances were also found by Thielsch et al. (2017) in the DLSC and mitochondrial capture has been detected in other Daphnia species (Marková et al. 2013). It is an interesting phenomenon in the DLSC that merits to be further investigated in the future with broader sampling.

In EIC, both pelagic samples and resting eggs were analyzed. Genotypes sampled alive from the water column were all inferred to be admixed to various degrees, three resting egg samples were also admixed, and the remaining five were assigned to either one of the two parental species. We conducted the ancestry painting approach on all EIC individuals; the fixed sites heterozygosity of the individuals categorized as nonadmixed in ADMIXTURE was indeed near zero (supplementary table S7, Supplementary Material online). Such high abundances of $D$. galeata $\times$ longispina hybrid resting eggs in periods of rapidly changing environmental conditions (i.e., eutrophication) have also been recorded in Lake Constance (Brede et al. 2009). The high frequency of $D$. galeata $\times$ longispina hybrids observed here might be due to similar ecological history: the lake Eichbaumsee was created through sand excavation for construction work around $\sim 40$ years ago and is characterized by extreme eutrophication and even hypertrophy that could not be remediated. The presence of later-generation hybrids and backcrosses with $D$. galeata and $D$. longispina and short ancestry tract length suggest that both species have been present and hybridizing for most of the lake's short history, or even that it was colonized by individuals of hybrid origin. However, we only obtained contemporary samples for EIC and analysis of resting eggs from sediment cores are needed to distinguish between the two hypotheses.
In $\mathrm{SE}$, diversity is high, both in terms of species combinations in admixed individuals and in terms of degrees of introgression. Although we analyzed eggs from sediments cores, they originated from the first centimeters and there is, therefore, no clear temporal pattern that separates the different hybrid combinations found here. Strikingly, although SE and DOB are geographically very close to each other $(\sim 10 \mathrm{~km})$, and dispersal of resting eggs through, for example, waterfowl or storms would be possible (Figuerola et al. 2005; Pietrzak and Slusarczyk 2006; Frisch et al. 2007), the Daphnia communities are quite different. This might be due to their different eutrophication levels, reflect the fact that initial colonization was followed by the establishment of different species, or a combination of both. The observed diversity at such a small spatial scale underlines the mosaic nature of freshwater habitats and the usefulness of approaches including many populations to fully understand genetic diversity arising from colonization and hybridization events in the DLSC.

Previous studies using mitochondrial and few nuclear markers (Thielsch et al. 2012; Alric et al. 2016) were able to categorize hybrids into F1, F2, and backcrosses. However, due to the low resolution of the used markers, further categorizing and above all identification of genome-wide breaking points was not possible at the time. The $D$. galeata reference genome and resequencing data offer now a much higher resolution to assess later generation hybrids and patterns across the genome. In general, hybrids identified in this study seem to have a complex history of multiple generations of hybridization and backcrossing with both parental species that we are not able to detangle using only ancestry paintings. The local ancestry inference revealed that the average ancestry tract length for $D$. galeata $\times$ longispina hybrids from EIC and SE is shorter than those for $D$. galeata $\times$ cucullata hybrids. There are several explanations for the observed pattern. One is that more generations of recombination led to shorter introgressed tracts, and the $D$. galeata $\times$ longispina hybrids are therefore the result of a greater number of sexual generations than the $D$. galeata $\times$ cucullata hybrids. The genomic mosaic of ancestry segments for all hybrid individuals is also characterized by multiple breakpoints within the same scaffolds, which is only possible after multiple generations of recombination. However, data on genome-wide recombination rates and selection are needed to reach solid conclusions about the correlation between tract length and age of the hybridization event in the individual's ancestors. Alternatively, reproductive isolation might be lower between $D$. galeata and $D$. longispina than between $D$. galeata and D. cucullata, thus leading to faster introgression in the former case.

As evidenced by the comparison of genomic windows of higher divergence between species pairs, the introgression pattern is not random: a given region exhibiting high $F_{\mathrm{ST}}$ values between the $D$. galeata and $D$. longispina genotypes is also likely to show similarly high $F_{S T}$ values in the $D$. galeata/ 
D. cucullata pair. Further, some parts of the genome seem to be effectively shielded from introgression. About a quarter of all genes $(4,136)$ are in regions that are highly differentiated between at least two species and about 5\% (859) in parts of the genome that are isolated among all three species of the complex. This is much more than expected by chance (supplementary fig. S3, Supplementary Material online) and is thus likely due to selection against introgression. It seems plausible to search among these for genes that conserve the specific identity of the involved taxa, despite incomplete reproductive isolation. Genes responsible for the observed ecological divergence among the taxa (Schwenk et al. 2000) or genetic incompatibilities are most likely candidates to be found in the observed divergent regions. Given the ancient divergence, the speciation process in the DLSC might have attained a selection-migration-drift equilibrium, for which there is growing empirical evidence in other species like stick insects (Riesch et al. 2017), flycatchers (Burri et al. 2015), and nonbiting midges (Schreiber and Pfenninger 2021). However, the current snapshot could equally likely be a consequence of one or several pulses of hybridization. To assess the stability of the equilibrium, data showing that the introgression/selection process is ongoing and constant across an extended period of time would be required and Daphnia offers the unique opportunity to go back in time to test these alternative hypotheses.

\section{New Evidence for Cytonuclear Discordance}

The genome-wide perspective also elucidated discordance between nuclear and mitochondrial patterns. The phylogeny based on mitochondrial genomes conforms to previously inferred relationships in the DLSC and suggests $D$. galeata and $D$. cucullata are sister species, with $D$. longispina as an outgroup (Adamowicz et al. 2009; Petrusek et al. 2012). However, several of our analyses based on nuclear SNPs challenge this view and suggest different evolutionary histories for mitochondrial and nuclear genomes. The ancestry painting approach relies on the identification of fixed sites for species in a pairwise comparison. More sites were found to be fixed between $D$. galeata and $D$. cucullata $(715,438)$ than between $D$. galeata and $D$. longispina $(335,052)$, which implies a greater divergence between members of the former pair. Further, $F_{\text {ST }}$ values were on an average higher between $D$. galeata and $D$. longispina (0.274) than between $D$. galeata and $D$. cucullata (0.364). Reports of cytonuclear discordance are common both in plants (Huang et al. 2014; Stephens et al. 2015; Folk et al. 2017; Lee-Yaw et al. 2019) and animals (Llopart et al. 2014; Melo-Ferreira et al. 2014; Sarver et al. 2021). Several processes can lead to this discordance among closely related species: incomplete lineage sorting causing phylogenetic reconstructions based on mitochondrial markers to differ from the true phylogeny of the taxa, or selection causing the fixation of different mitochondrial genomes in different places from standing variation within species (Barrett and Schluter 2008). Alternatively, cytonuclear discordance may reflect hybridization between species and cytoplasmic introgression, accompanied or not by selection (reviewed in Sloan et al. [2017]). The latter explanation would be quite conceivable in the DLSC.

\section{Conclusion}

We here provide the first high-quality resources to study genome-wide patterns of divergence in the DLSC, an ecologically important taxon in European freshwater habitats. By quantifying intra- and interspecific diversity, we provide a first glimpse into introgressive hybridization and lay the ground for further studies aiming at understanding how species boundaries are maintained in the face of gene flow.

Unlike for $D$. pulex and $D$. magna, no linkage groups are known for any species of the DLSC. Hi-C sequencing data will be added in the future to order scaffolds into larger, potentially chromosome-scale scaffolds. Such an approach holds promise in a species complex were laboratory crossings for F2 panels and traditional mapping are nearly impossible. This will allow discovering structural variants, identifying recombination breakpoints along each chromosome and thus provide a deeper understanding of the introgression patterns observed here. The functional role of genes in the regions of high divergence uncovered through this first analysis is yet unclear and will be addressed in future studies.

Finally, wider sampling, with the inclusion of more populations as well as more members of the species complex, and the reconstruction of a nuclear based phylogeny are necessary to reach conclusions about the species relationships and eventually identify the causes of the pattern uncovered here.

\section{Materials and Methods}

\section{Sampling}

The clonal line used for genome sequencing and assembly, M5, was hatched from a resting egg isolated from the upper layers (first $5 \mathrm{~cm}$, corresponding to the years 2000-2010) of a sediment core taken in Lake Müggelsee in 2010. Further, single genotypes representing the parental species from various locations were used in this study, henceforth "parental species genotypes." Most of them were established from individuals sampled from the water column and are still maintained through asexual reproduction as monoclonal cultures in the laboratory. Thus, all individuals of a clonal line are the same genotype and can be pooled to achieve sufficient amounts of genomic DNA. The species identity for these genotypes was established through a combination of methods: morphology, mitochondrial sequences, and nuclear markers.

Sediment cores were collected from Dobersdorfer See (DOB), Selenter See (SE), and Arendsee (AR), Germany using 
a gravity corer (Uwitec, Mondsee, AT) (supplementary table S4, Supplementary Material online). Samples were taken from the deepest part of the lakes to minimize past disturbance of the sediment. Cores were cut horizontally into $1 \mathrm{~cm}$ layers and the layers were stored at $4{ }^{\circ} \mathrm{C}$ in the dark to prevent hatching. Sediment rate of the three lakes was determined using radioisotope dating $\left({ }^{137} \mathrm{Cs}\right.$ and $\left.{ }^{210} \mathrm{~Pb}\right)$.

In addition, lake sediment from the shoreline of Eichbaumsee (EIC) (supplementary table S4, Supplementary Material online), Germany was collected by hand and stored at $4{ }^{\circ} \mathrm{C}$. The exact age of the sediment is unknown but the upper layers most likely contain recent eggs from the last few years. Zooplankton samples were taken from Eichbaumsee using a plankton net (mesh size $150 \mu \mathrm{m}$ ) from which six Daphnia clonal lines were established in a laboratory setting with artificial medium (Aachener Daphnien Medium, ADaM Klüttgen et al. 1994).

All sampling locations are plotted in figure $1 A$ and information on all samples is provided in supplementary table $\mathbf{S}$, Supplementary Material online.

\section{Genome Sequencing}

\section{DNA Extraction for Genome Sequencing with Illumina and PacBio}

DNA was extracted from around 60 clonal M5 individuals collected from batch cultures maintained in ADaM, and fed with the algae Acutodesmus obliquus, cultivated in medium modified after (Zehnder and Gorham 1960). Extraction was conducted following a phenol chloroform-based protocol with an RNase step and subsequently sequenced on an Illumina HiSeq4000 at BGI China. Additionally, tissue samples with around 3,000 individuals were sent to BGI for DNA extraction and PacBio sequencing.

\section{Resequencing (Population Genomics Approach) DNA Extraction from Batch Cultures for Resequencing}

For clonal lines used as reference for the parental species, individuals were raised in batch cultures and treated with antibiotics prior to collection and storage at -20 or $-80^{\circ} \mathrm{C}$. DNA was extracted with either a phenol chloroform method, a (modified) CTAB protocol or a rapid desalting method (MasterPure Complete DNA and RNA Purification Kit; Lucigen Corporation).

Total genomic DNA was isolated from 20 pooled adult Daphnia for each of the five EIC clonal lines using a CTAB extraction method (Doyle and Doyle 1987).

\section{Whole-Genome Amplification on Resting Eggs for Resequencing}

To isolate Daphnia resting eggs from the sediment each sediment layer was sieved using a sieve with $125 \mu \mathrm{m}$ mesh size and small amounts of the remaining sediment were resuspended in distilled water. Ephippia were eye spotted under a stereomicroscope, counted, and transferred to $1.5 \mathrm{ml}$ tubes. The water was removed and ephippia stored at $-20^{\circ} \mathrm{C}$ in the dark until further analysis.

The ephippia were then opened under a binocular with insect needles and tweezers previously treated under a clean bench (UV sterilization) and with DNase away (Thermo Fisher). Eggs that were already damaged, had an uneven shape or were orange, which is evidence for degradation, were discarded. The resting egg separated from the ephippial casing washed in $15 \mu \mathrm{l}$ sterile $1 \times$ PBS and then transferred in $1 \mu \mathrm{l} 1 \times$ PBS to a new tube with $2 \mu$ fresh $1 \times$ PBS. The isolated eggs were stored at $-80^{\circ} \mathrm{C}$ at least overnight.

For WGA of single eggs, the REPLI-g Mini Kit (Qiagen) was used. This kit is enabling unbiased amplification of genomic loci via Multiple Displacement Amplification. The isolated resting eggs were thawed on ice and the whole genome was amplified following the manufacturer's protocol for amplification of genomic DNA from blood or cells. Briefly, denaturation buffer was added to the prepared resting eggs in $3 \mu \mathrm{l}$ $1 \times$ PBS and amplified by REPLI-g Mini DNA Polymerase under isothermal conditions for 16 hours.

The amplified product was quantified on a Nanodrop spectrophotometer (Thermo Fisher) and with a Qubit Fluorometer (Thermo Fisher). Successful amplifications were purified with 0.4× Agencourt AMPure XP magnetic beads (Beckman Coulter) to remove small fragments and eluted in $60 \mu \mathrm{l} 1 \times$ TE buffer.

Fragments of the mitochondrial gene $16 \mathrm{~S}$ rRNA gene were amplified to check successful amplification of Daphnia DNA using the universal cladoceran primers S1 and S2 (Schwenk et al. 1998) and a low presence of bacterial DNA using universal primers for the bacterial $16 \mathrm{~S}$ rDNA gene (Nadkarni et al. 2002). Only samples with a successful amplification of the Daphnia 165 fragment and low amplification of the bacterial 165 fragment indicating low bacterial contamination were used for sequencing steps.

\section{Library Preparation and Sequencing of Resequencing Samples}

After quantification and quality control of the DNA using Nanodrop and Qubit instruments, libraries were prepared either directly in-house with the NEBNext Ultra II DNA Library Prep Kit for Illumina (New England Biolabs), or at the sequencing company Novogene (Cambridge, UK). Resequencing (paired-end 150-bp reads) was then performed either at Novogene (UK) Company Limited or the Functional Genomics Center (ETH Zurich and University of Zurich) on Illumina NovaSeq 6000 and HiSeq4000 instruments.

Details on the procedure used for each sample are provided in supplementary table $\mathbf{S 2}$, Supplementary Material online. 


\section{Genome Assembly and Annotation}

We provide here a summarized version of the procedure used to assemble and annotate the genome. Details can be found in supplementary methods, Supplementary Material online.

\section{Raw Data QC}

Illumina reads were trimmed and the adapter removed using a combination of Trimmomatic 0.38 (Bolger et al. 2014), FastQC 0.11.7 (Andrews 2010) and MultiQC 1.6 (Ewels et al. 2016) within autotrim 0.6.1 (Waldvogel et al. 2018). To filter out reads possibly originating from contamination from known sources (see below), a FastQ Screen like approach was chosen. In brief, the reads are separated by results of mapping behavior to different genomes. Positive controls consisted of genome data for other Daphnia species (dmagna-v2.4 and Daphnia_pulex_PA42_v3.0, see supplementary methods, Supplementary Material online, for accession numbers), and negative control, that is, sequences deemed undesirable for genome assembly consisted of genome data from human, bacteria, viruses, and the algae used to feed the batch cultures. The resulting database comprised 108,163 sequences (total sequence space $42.2 \mathrm{~Gb}$ ). Both Illumina reads and PacBio subreads were mapped against the database with NextGenMap (Sedlazeck et al. 2013) and minimap2 (Li 2018), respectively.

Reads did only pass the filtering if they either did not map to the database at all or had at least one hit against one of the two Daphnia genomes. Supplementary table S2, Supplementary Material online gives an overview of the effect of different filtering steps.

\section{Assembly and Contamination Screening}

All paired and unpaired contamination filtered Illumina reads as well as the contamination filtered PacBio reads were used as input for RA 0.2.1 (https://github.com/rvaser/ra, last accessed November 2021). Blobtools 1.0 (Laetsch and Blaxter 2017) was used to screen the resulting assembly for possible unidentified contamination in the hybrid assembly. Briefly, bwa mem 0.7.17 (Li 2013) was used to map Illumina reads back to the assembly and taxonomic assignment was done by sequence similarity search with BlastN 2.9.0+ (Camacho et al. 2009). Contamination with different bacteria was clearly identifiable, and contigs with coverage below $10 \times$ and/or GC content above $50 \%$ were removed. Additionally, PacBio reads mapping to these contigs were removed to minimize false scaffolding in further steps. The contig corresponding to the mitochondrial genome was identified after a BLAST search against available mitochondrial genomes for this species and removed from the assembly.

\section{Scaffolding and Gap Closing}

The blobtools filtered PacBio reads were used for scaffolding and gapclosing, which was conducted in three iterations. Each iteration consisted of a scaffolding step with SSPACE LongRead 1-1 (Boetzer and Pirovano 2014), a gap closing step with LR Gapcloser (https://github.com/CAFS-bioinformatics/LR_Gapcloser; commit 156381a, last accessed November 2021), and a step to polish former gap parts with short reads using bwa mem 0.7.17-r1188 and Pilon 1.23 (Walker et al. 2014) in a pipeline developed to this effect, wtdbg2-racon-pilon.pl $\quad 0.4 \quad$ (https://github.com/schellt/ wtdbg2-racon-pilon, last accessed November 2021).

\section{Assembly Quality Assessment}

Contiguity was analyzed with Quast 5.0.2 (Gurevich et al. 2013) at different stages of the assembly process. Further, mapping rate, coverage, and insert size distribution were assessed by mapping Illumina and PacBio reads with bwa mem and Minimap 2.17 respectively. To show absence of contamination in the assembly blobtools was ran as above. The genome size was estimated by dividing the mapped nucleotides by the mode of the coverage distribution of the Illumina reads by backmap 0.3 (https://github.com/schellt/ backmap, last accessed January 2021), resulting in $156.86 \mathrm{Mb}$ (with the obtained assembly length amounting to $85 \%$ of this estimated length). Additionally, the genome size was estimated using a k-mer based approach by creating a histogram from raw Illumina reads with Jellyfish 1.1.12 (Marçais and Kingsford 2011) and running the GenomeScope web application (http://qb.cshl.edu/genomescope/, last accessed November 2021) resulting in a genome size estimate of $150.6 \mathrm{Mb}$.

Completeness in terms of single copy core orthologs of the final scaffolds was assessed with BUSCO 3.0.2 (Simão et al. 2015), using the Arthropoda set (odb9).

\section{Genome Annotation}

RepeatModeler 2.0 (Smit and Hubley 2015) was run to identify $D$. galeata specific repeats. The 1,115 obtained repeat families were combined with 237 D. pulex and one $D$. pulicaria repeat sequences from RepBase release 20181026 to create the final repeat library. The genome assembly was then soft masked with RepeatMasker 4.1.0 (Smit et al. 2013-2015), resulting in $21.9 \%$ of the assembly being masked.

Gene prediction models were produced with Augustus 3.3.2 (Stanke et al. 2008), GeneMark ET 4.48_3.60_lic (Lomsadze et al. 2005), and SNAP 2006-07-28 (Korf 2004). The Augustus model was based on the soft masked assembly and the D. galeata transcriptome (HAFN01.1, Huylmans et al. 2016). The GeneMark model was obtained by first mapping 
trimmed RNAseq reads to the assembly with HISAT 2.1.0 (Kim et al. 2019) and then processing the resulting bam file with bam2hints and filterlntronsFindStrand.pl from Augustus to create a gff file with possible introns, which was finally fed into GeneMark.

The structural annotation was conducted in MAKER 2.31.10 (Holt and Yandell 2011). Briefly, the unmasked genome assembly, the species own transcriptome assembly as ESTs, the complete Swiss-Prot 2019_10 (UniProt Consortium 2019) and the protein sequences resulting from $D$. magna (Lee et al. 2019), as well as D. pulex (Ye et al. 2017) genome annotations as protein evidence, were used as input for MAKER. In total, three iterations of MAKER with retraining of the Augustus and SNAP model in between the iterations were conducted.

The quality of the structural annotation was assessed by comparing values as number of genes, gene space, etc. to existing annotations for other Daphnia genomes. Furthermore, core orthologs from BUSCO's Arthropoda (odb9) set and conserved domain arrangements from the Arthropoda reference set of DOGMA 3.4 (Dohmen et al. 2016) were searched in the annotated protein set.

The functional annotation was conducted using InterProScan 5.39-77.0 (Jones et al. 2014) as well as a BLAST against the Swiss-Prot 2019_10.

\section{Population Samples \\ Raw Data QC and Contamination Check}

The quality of raw reads was checked using FastQC v0.11.5. Adapter trimming and quality filtering were performed using Trimmomatic v0.36 with the following parameters: ILLUMINACLIP: TruSeq3-PE.fa: 2:30:10 TRAILING: 20 SLIDINGWINDOW: 4:15 MINLEN: 70. For samples sequenced on a NovaSeq6000 instrument and presenting a typical polyG tail, the program fastp (Chen et al. 2018) was used for trimming as well. To assess contamination in the WGA samples FastQ Screen v0.14.0 with the bwa mapping option was used (Wingett and Andrews 2018). A custom database was built to map trimmed reads against possible contaminants that included general common contaminants such as Homo sapiens, the UniVec reference database, a bacterial and a viral reference set as well as the $D$. galeata genome and Acutodesmus obliquus draft genome (see supplementary methods, Supplementary Material online, for accession numbers). Samples with $<25 \%$ reads mapped to the $D$. galeata genome (and 25\% contamination) were excluded from further analysis because the whole amplification of the resting egg most likely failed.

\section{Mapping to Reference Genome and Variant Calling}

The variant calling was performed within the Genome Analysis Toolkit (GATK v4.1.4.0; McKenna et al. 2010) program according to GATK4 best practices (Van der Auwera et al. 2013). The trimmed reads were mapped to the $D$. galeata genome using the BWA-MEM algorithm in BWA v0.7.17 with the -M parameter and adding read group identifiers for Picard compatibility (Li and Durbin 2009). PCR duplicates were marked and filtered out in the BAM file using Picard v2.21.1 (http://broadinstitute.github.io/picard/, last accessed November 2021).

To call variants for each sample GATK HaplotypeCaller in GATK was used with the -emitRefConfidence GVCF option resulting in a genomic variant call format (gVCF) file with information on each position for each individual (Poplin et al. 2018). All gVCF files were consolidated using CombineGVCFs and joint genotyping was performed with GenotypeGVCFs. The VCF file was filtered to include only SNPs and hard filtering was performed to remove variants with a QualByDepth $<10$, StrandOddsRatio $>3$, FisherStrand $>60$, mapping quality $<40$, MappingQualityRankSumTest $<-8$, and ReadPosRank SumTest $<-5$.

Subsequently, we removed sites with either very high coverage $(>450)$ or for which genotypes were missing for more than $20 \%$ of the individuals using VCFtools v0.1.5 (Danecek et al. 2011). The final SNP data set for downstream analyses included 3,240,339 SNPs across the 49 samples.

In addition, GenotypeGVCFs was run with the -includenon-variant-sites option to output all variant as well as invariant genotyped sites. The final invariant data set included $127,530,229$ sites after removing indels and multiallelic sites with BCFtools v1.9 (Li 2011) and is used for population genomic analysis to be able to calculate the total number of genotyped sites (variant and invariant) within a genomic window.

As we mapped all different species to the reference $D$. galeata genome, we assessed possible reference bias by checking the distribution of reference and alternative alleles observed at heterozygous genotypes based on Pinsky (2021). We pooled all genotypes which were unambiguously assigned to either of the parental species clusters D. galeata, D. cucullata, and $D$. longispina as was done for the population genomic parameters (supplementary table S2, Supplementary Material online) or classified as hybrids using ADMIXTURE inference. Without reference bias, we would expect that in heterozygous genotypes the reference and the alternative allele are on an average represented by $50 \%$ of the reads. An indication of reference bias would be that the $D$. galeata reference allele would be more frequent.

\section{Phylogenetic and Population Genetics Inferences \\ Mitochondrial Genome Assemblies and Phylogenetic Analyses}

All reads were used to produce mitochondrial genome assemblies using the "de novo assembly" and "find mitochondrial scaffold" modules provided in MitoZ v2.4 with default 
settings (Meng et al. 2019). For some samples, this was not sufficient and we used two approaches to recover a complete mitogenome: either the mitochondrial baiting and iterative mapping implemented in MITObim v1.9.1 (Hahn et al. 2013) with the $D$. galeata mitochondrial reference genome or the modified baiting and iterative mapping in GetOrganelle v1.7.1 (Jin et al. 2020) with the animal database and k-mer values set to $21,45,65,85$, and 105 . The procedure used for each data set is given in supplementary table S5, Supplementary Material online.

We annotated the mitochondrial genome assemblies with the mitochondrial annotation web server MITOS2 (Bernt et al. 2013) using the mitochondrial codon code 05 for invertebrates. Automated genome annotation identified 13 PCGs, two rRNA genes, and 22 transfer RNA genes (tRNAs). Initially, the mitochondrial genes (PCGs and rRNAs, supplementary table S6, Supplementary Material online) were individually aligned with MUSCLE v3.8.1551 (Edgar 2004) and visually checked for their quality. The mitochondrial genome assemblies with discrepancies, that is, a lot of missing data and/or split features were excluded from further analysis. The final data set (supplementary table S6, Supplementary Material online) included 44 mitochondrial genomes from this study and the previously published mitochondrial genome of Daphnia laevis (Martins Ribeiro et al. 2019, accession number: NC_045243.1). The mitochondrial genes of the final data set were individually realigned with MUSCLE v3.8.1551 (Edgar 2004) and MACSE V2.05 (Ranwez et al. 2018) and concatenated into a mitochondrial DNA matrix (supplementary table S6, Supplementary Material online) using SequenceMatrix v1.8.1 (Vaidya et al. 2011). During this step, we used MACSE v2.05 to realign PCG genes keeping the information about codon position (gene partitioning) and to remove STOP codons. The final data set consisted of the concatenation matrix of the 13 PCGs and the two structural rRNA genes. With this alignment, phylogenetic trees were reconstructed using IQ-TREE v1.6.12 (Nguyen et al. 2015). We initially partitioned the alignment into a full partition model, that is, each gene and all three codon positions for PCGs, and then ran IQ-TREE with partition analyses (-spp, Chernomor et al. 2016), ModelFinder (- m MFP+MERGE, Kalyaanamoorthy et al. 2017) and 10,000 ultrafast bootstrap (-bb 10,000, Hoang et al. 2018) and SH-like approximate likelihood ratio test (-alrt 10,000, Guindon et al. 2010) replicates. The resulting trees were visualized in $\mathrm{R}$ ( $\mathrm{R}$ Core Team 2017) using the multifunctional phylogenetics package phytools (Revell 2012).

\section{Ancestry and Population Structure}

A principal component analysis was conducted in R v3.6.2 ( $R$ Core Team 2017) with the package SNPRelate v1.20.1 (Zheng et al. 2012). Linkage disequilibrium (LD) was calculated within a 500-kb sliding window and LD-pruned for $r^{2}$ values $>0.5$ before conducting the PCA for all sites using the snpgdsPCA function with default settings. The relative large $L D$ value was chosen because clonal reproduction and the overlap of generations due to diapause leads to increased LD in Daphnia (Brede et al. 2009).

Genetic admixture was estimated using ADMIXTURE v1.3.0 (Alexander and Lange 2011). The SNP set VCF file was converted to BED format using plink v1.90b6.13 (Chang et al. 2015). The log-likelihood values were estimated for one to five genetic clusters ( $K$ ) of ancestral populations and admixture analysis were run for the most appropriate $K$ value with 10-fold cross-validation. We also conducted the PCA and Admixture analysis using PCAngsd implemented in ANGSD and NgsAdmix, respectively (Korneliussen et al. 2014) to take genotype likelihoods into account (details in supplementary methods, Supplementary Material online). The results did not differ substantially and are shown in supplementary figure S3, Supplementary Material online.

However, using such a population genetic clustering approach to estimate ancestry coefficients is not directly equivalent to the proportion of hybrid ancestry in each individual and should be interpreted with caution (Lawson et al. 2018; Kong and Kubatko 2021). The results of the ADMIXTURE analysis suggested that the data set included hybrids between $D$. longispina and D. galeata as well as $D$. cucullata and $D$. galeata. We then followed the "ancestry painting" procedure outlined in Barth et al. (2020) and Runemark, Trier, et al. (2018), and classified sites according to their $F_{\text {ST }}$ values when comparing parental species sets. Unlike the PCA and the admixture analysis, this approach requires the user to define parental genotypes; the individuals belonging to these sets are indicated with stars in figure $1 C$. Fixed sites are those where a specific allele is fixed in all individuals belonging to one parental species and another allele fixed in the other parental species. To show the ancestry of the hybrid individuals each fixed site was plotted in an "ancestry painting" if at least $80 \%$ of genotypes were complete using available ruby scripts (https://github.com/mmatschiner/tutorials/tree/master/analysis_of_introgression_with_snp_data, last accessed November 2021). These scripts calculate the heterozygosity of each individual and visualize regions that are possibly affected by introgression. The mitochondrial genome assembly from each individual was used to determine the maternal species and the proportion of the genome derived from the maternal species was then calculated for each hybrid. For gal $\times$ cuc hybrids the hybrid index scale ranges from 0 (gal) to 1 (cuc) and for gal $\times$ long hybrids from 0 (long) to 1 (gal).

\section{Window-Based Population Parameters}

To assess genome-wide genetic differentiation between the clusters identified with admixture, we calculated nucleotide diversity $(\pi)$, between-taxon differentiation $\left(F_{S T}\right)$, and between-taxon divergence $\left(d_{x y}\right)$ using the Python script 


\section{Supplementary Material}

Supplementary data are available at Genome Biology and Evolution online.

\section{Acknowledgments}

We thank LOEWE-TBG for providing sequencing funds. T.H. and M.M. were supported by the grant "SeeWandel: Life in Lake Constance - the past, present and future" within the framework of the Interreg $V$ programme "Alpenrhein-BodenseeHochrhein (Germany/Austria/Switzerland/Liechtenstein)," which funds are provided by the European Regional Development Fund as well as the Swiss Confederation and cantons. M.M. was supported by the Austrian Science Fund (FWF): P29667B25 and J 3774. The funders had no role in study design, data collection and analysis, decision to publish, or preparation of the manuscript. The computational results presented here have been achieved in (part) using the LEO HPC infrastructure of the University of Innsbruck. Some of the data produced and analyzed in this paper were generated in collaboration with the Genetic Diversity Centre (GDC), ETH Zurich. We thank Jae-Seong Lee and Zhiqiang Ye for giving us access to genome annotations for D. magna (SK strain) and D. pulex (PA42 strain), respectively. Miklós Bálint provided sediment samples and isotope data for the Arendsee lake. We thank Michael Matschiner for his help with ancestry painting and two anonymous reviewers for their comments on a previous version of the manuscript.

\section{Author Contributions}

M.C., J.N., A.T., S.R.D., and M.M. performed the sampling and wet lab work. T.S., J.N., and A.T. conducted the genome assembly and annotation. J.N. conducted the population genomic analysis and mitochondrial genome reconstruction. T.H. and M.M. conducted the phylogenetic analyses. J.N., T.S., M.C., M.P., M.M., and T.H. wrote the manuscript, and all authors edited and contributed to the final version. All authors gave final approval for preprint deposition and publication.

\section{Data Availability}

Genome assembly, annotation, and read data (Illumina and PacBio) for the genotype M5 are stored under accession number PRJEB42807. Short-read data from resequencing are available in the European Nucleotide Archive under accession numbers ERS5080327-ERS5080375, ERS4993274, and ERS4993282. The annotation and genome assembly used in the present analysis are deposited in Zenodo (doi:10.5281/ zenodo.4479324), together with supplementary information on the mitochondrial tree (alignment file). 


\section{Literature Cited}

Abbott R, et al. 2013. Hybridization and speciation. J Evol Biol. 26(2):229-246.

Adamowicz SJ, Petrusek A, Colbourne JK, Hebert PDN, Witt JDS. 2009. The scale of divergence: a phylogenetic appraisal of intercontinental allopatric speciation in a passively dispersed freshwater zooplankton genus. Mol Phylogenet Evol. 50(3):423-436.

Alexander DH, Lange K. 2011. Enhancements to the ADMIXTURE algorithm for individual ancestry estimation. BMC Bioinformatics 12:246.

Alric $B$, et al. 2016. Local human pressures influence gene flow in a hybridizing Daphnia species complex. J Evol Biol. 29(4):720-735.

Andrews S. 2010. FastQC: a quality control tool for high throughput sequence data. Available from: https://www.bioinformatics.babraham.ac.uk/projects/fastqd. Accessed November 2021.

Arnold ML, Martin NH. 2009. Adaptation by introgression. J Biol. 8:82.

Barrett RD, Schluter D. 2008. Adaptation from standing genetic variation. Trends Ecol. Evol. 23(1):38-44.

Barth JM, et al. 2020. Stable species boundaries despite ten million years of hybridization in tropical eels. Nat Commun. 11:1-13.

Barton NH, Hewitt GM. 1985. Analysis of hybrid zones. Annu Rev Ecol Syst. 16(1):113-148.

Beaton MJ, Hebert PD. 2004. Variation in chromosome numbers of Daphnia (Crustacea, Cladocera). Hereditas 120(3):275-279.

Bernt M, et al. 2013. MITOS: improved de novo metazoan mitochondrial genome annotation. Mol Phylogenet Evol. 69(2):313-319.

Billiones R, Brehm M, Klee J, Schwenk K. 2004. Genetic identification of Hyalodaphnia species and interspecific hybrids. Hydrobiologia 526(1):43-53.

Boetzer M, Pirovano W. 2014. SSPACE-LongRead: scaffolding bacterial draft genomes using long read sequence information. BMC Bioinformatics 15:211.

Bolger AM, Lohse M, Usadel B. 2014. Trimmomatic: a flexible trimmer for Illumina sequence data. Bioinformatics 30(15):2114-2120.

Brede $\mathrm{N}$, et al. 2006. Microsatellite markers for European Daphnia. Mol Ecol Notes. 6(2):536-539.

Brede $\mathrm{N}$, et al. 2009. The impact of human-made ecological changes on the genetic architecture of Daphnia species. Proc Natl Acad Sci U S A. 106(12):4758-4763.

Browning BL, Browning SR. 2009. A unified approach to genotype imputation and haplotype-phase inference for large data sets of trios and unrelated individuals. Am J Hum Genet. 84(2):210-223.

Burri $\mathrm{R}$, et al. 2015. Linked selection and recombination rate variation drive the evolution of the genomic landscape of differentiation across the speciation continuum of Ficedula flycatchers. Genome Res. 25(11):1656-1665.

Butlin RK, et al. 2014. Parallel evolution of local adaptation and reproductive isolation in the face of gene flow. Evolution 68(4):935-949.

Camacho C, et al. 2009. BLAST+: architecture and applications. BMC Bioinformatics 10:421.

Canestrelli D, et al. 2017. Climate change promotes hybridisation between deeply divergent species. PeerJ 5:e3072.

Chang CC, et al. 2015. Second-generation PLINK: rising to the challenge of larger and richer datasets. Gigascience 4(1):7.

Chen S, Zhou Y, Chen Y, Gu J. 2018. fastp: an ultra-fast all-in-one FASTQ preprocessor. Bioinformatics 34(17):i884-i890.

Chernomor O, Von Haeseler A, Minh BQ. 2016. Terrace aware data structure for phylogenomic inference from supermatrices. Syst Biol. 65(6):997-1008

Colbourne JK, et al. 2011. The ecoresponsive genome of Daphnia pulex. Science 331(6017):555-561.

Cordellier M, Wojewodzic MW, Wessels M, Kuster CJ, Von Elert E. 2021. Next-generation sequencing of DNA from resting eggs: signatures of eutrophication in a lake's sediment. Zoology (Jena) 145:125895.
Cornetti L, Fields PD, Van Damme K, Ebert D. 2019. A fossil-calibrated phylogenomic analysis of Daphnia and the Daphniidae. Mol Phylogenet Evol. 137:250-262.

Cousyn C, et al. 2001. Rapid, local adaptation of zooplankton behavior to changes in predation pressure in the absence of neutral genetic changes. Proc Natl Acad Sci U S A. 98(11):6256-6260.

Danecek P, et al. 2011. The variant call format and VCFtools. Bioinformatics 27(15):2156-2158.

Dias-Alves T, Mairal J, Blum MG. 2018. Loter: a software package to infer local ancestry for a wide range of species. Mol Biol Evol. 35(9):2318-2326.

Dlouha S, et al. 2010. Identifying hybridizing taxa within the Daphnia longispina species complex: a comparison of genetic methods and phenotypic approaches. Hydrobiologia 643:107-122.

Doellman MM, et al. 2018. Genomic differentiation during speciationwith-gene-flow: comparing geographic and host-related variation in divergent life history adaptation in Rhagoletis pomonella. Genes 9(5):262.

Dohmen E, Kremer LP, Bornberg-Bauer E, Kemena C. 2016. DOGMA: domain-based transcriptome and proteome quality assessment. Bioinformatics 32(17):2577-2581.

Doyle JJ, Doyle JL. 1987. A rapid DNA isolation procedure for small quantities of fresh leaf tissue. Phytochem Bull. 19:11-15.

Dziuba MK, et al. 2020. Temperature increase altered Daphnia community structure in artificially heated lakes: a potential scenario for a warmer future. Sci Rep. 10(1):13956.

Edgar RC. 2004. MUSCLE: multiple sequence alignment with high accuracy and high throughput. Nucleic Acids Res. 32(5):1792-1797.

Ewels P, Magnusson M, Lundin S, Käller M. 2016. MultiQC: summarize analysis results for multiple tools and samples in a single report. Bioinformatics 32(19):3047-3048.

Figuerola J, Green AJ, Michot TC. 2005. Invertebrate eggs can fly: evidence of waterfowl-mediated gene flow in aquatic invertebrates. Am Nat. 165(2):274-280.

Flaxman SM, Wacholder AC, Feder JL, Nosil P. 2014. Theoretical models of the influence of genomic architecture on the dynamics of speciation. Mol Ecol. 23(16):4074-4088.

Folk RA, Mandel JR, Freudenstein JV. 2017. Ancestral gene flow and parallel organellar genome capture result in extreme phylogenomic discord in a lineage of angiosperms. Syst Biol. 66:320-337.

Fraïsse C, Roux C, Welch JJ, Bierne N. 2014. Gene-flow in a mosaic hybrid zone: is local introgression adaptive? Genetics 197(3):939-951.

Frisch D, et al. 2014. A millennial-scale chronicle of evolutionary responses to cultural eutrophication in Daphnia. Ecol Lett. 17(3):360-368.

Frisch D, Green AJ, Figuerola J. 2007. High dispersal capacity of a broad spectrum of aquatic invertebrates via waterbirds. Aquat Sci. 69(4):568-574.

Gannon JE, Stemberger RS. 1978. Zooplankton (especially Crustaceans and Rotifers) as indicators of water quality. Trans Am Microsc Soc. 97(1):16-35.

Gliwicz ZM. 1990. Why do cladocerans fail to control algal blooms? In: Gulati RD, Lammens EHRR, Meijer ML, van Donk E, editors. Biomanipulation tool for water management. Developments in Hydrobiology, vol 61. Dordrecht: Springer. p. 83-97.

Griebel J, Gießler S, Yin M, Wolinska J. 2016. Parental and hybrid Daphnia from the $D$. longispina complex: long-term dynamics in genetic structure and significance of overwintering modes. J Evol Biol. 29(4):810-823.

Guindon S, et al. 2010. New algorithms and methods to estimate maximum-likelihood phylogenies: assessing the performance of PhyML 3.0. Syst Biol. 59(3):307-321.

Gurevich A, Saveliev V, Vyahhi N, Tesler G. 2013. QUAST: quality assessment tool for genome assemblies. Bioinformatics 29(8):1072-1075. 
Hahn C, Bachmann L, Chevreux B. 2013. Reconstructing mitochondrial genomes directly from genomic next-generation sequencing reads-a baiting and iterative mapping approach. Nucleic Acids Res. 41(13):e129.

Harrison RG. 1986. Pattern and process in a narrow hybrid zone. Heredity 56(3):337-349

Herrmann M, Henning-Lucass N, Cordellier M, Schwenk K. 2017. A genotype-phenotype association approach to reveal thermal adaptation in Daphnia galeata. A genotype-phenotype association approach to reveal thermal adaptation in. J Exp Zool A Ecol Integr Physiol. 327(1):53-65.

Hoang DT, Chernomor O, Von Haeseler A, Minh BQ, Vinh LS. 2018. UFBoot2: improving the ultrafast bootstrap approximation. Mol Biol Evol. 35(2):518-522

Holt C, Yandell M. 2011. MAKER2: an annotation pipeline and genomedatabase management tool for second-generation genome projects. BMC Bioinformatics 12:491.

Huang DI, Hefer CA, Kolosova N, Douglas CJ, Cronk QC. 2014. Whole plastome sequencing reveals deep plastid divergence and cytonuclear discordance between closely related balsam poplars, Populus balsamifera and P. trichocarpa (Salicaceae). New Phytol. 204(3):693-703.

Huylmans AK, López Ezquerra A, Parsch J, Cordellier M. 2016. De novo transcriptome assembly and sex-biased gene expression in the cyclical parthenogenetic Daphnia galeata. Genome Biol Evol. 8(10):3120-3139.

Jin J-J, et al. 2020. GetOrganelle: a fast and versatile toolkit for accurate de novo assembly of organelle genomes. Genome Biol. 21(1):1-31.

Jones $P$, et al. 2014. InterProScan 5: genome-scale protein function classification. Bioinformatics 30(9):1236-1240.

Kaiser TS, von Haeseler A, Tessmar-Raible K, Heckel DG. 2021. Timing strains of the marine insect Clunio marinus diverged and persist with gene flow. Mol Ecol. 30(5):1264-1280.

Kalyaanamoorthy S, Minh BQ, Wong TK, von Haeseler A, Jermiin LS. 2017. ModelFinder: fast model selection for accurate phylogenetic estimates. Nat Methods. 14(6):587-589.

Keller B, Wolinska J, Manca M, Spaak P. 2008. Spatial, environmental and anthropogenic effects on the taxon composition of hybridizing Daphnia. Philos Trans R Soc Lond B Biol Sci. 363(1505):2943-2952.

Kim D, Paggi JM, Park C, Bennett C, Salzberg SL. 2019. Graph-based genome alignment and genotyping with HISAT2 and HISAT-genotype. Nat Biotechnol. 37(8):907-915.

Klüttgen B, Dülmer U, Engels M, Ratte HT. 1994. ADaM, an artificial freshwater for the culture of zooplankton. Water Res. 28(3):743-746.

Kong S, Kubatko LS. 2021. Comparative performance of popular methods for hybrid detection using genomic data. Syst Biol. 70(5):891-907.

Korf I. 2004. Gene finding in novel genomes. BMC Bioinformatics 5:59.

Korneliussen TS, Albrechtsen A, Nielsen R. 2014. ANGSD: analysis of next generation sequencing data. BMC Bioinformatics 15:356.

Lack JB, Weider $\amalg$, Jeyasingh PD. 2018. Whole genome amplification and sequencing of a Daphnia resting egg. Mol Ecol Resour. 18(1):1-10.

Laetsch DR, Blaxter ML. 2017. BlobTools: interrogation of genome assemblies. F1000Res. 6:1287.

Lampert W, Sommer U. 2007. Limnoecology: the ecology of lakes and streams. Oxford: Oxford University Press.

Lawson DJ, Van Dorp L, Falush D. 2018. A tutorial on how not to overinterpret STRUCTURE and ADMIXTURE bar plots. Nat Commun. 9:1-11.

Lee B-Y, et al. 2019. The genome of the freshwater water flea Daphnia magna: a potential use for freshwater molecular ecotoxicology. Aquat Toxicol. 210:69-84.

Lee-Yaw JA, Grassa CJ, Joly S, Andrew RL, Rieseberg LH. 2019. An evaluation of alternative explanations for widespread cytonuclear discordance in annual sunflowers (Helianthus). New Phytol. 221(1):515-526
Li H. 2011. A statistical framework for SNP calling, mutation discovery, association mapping and population genetical parameter estimation from sequencing data. Bioinformatics 27(21):2987-2993.

Li H. 2013. Aligning sequence reads, clone sequences and assembly contigs with BWA-MEM. arXiv: 1303.3997

Li H. 2018. Minimap2: pairwise alignment for nucleotide sequences. Bioinformatics 34(18):3094-3100.

Li H, Durbin R. 2009. Fast and accurate short read alignment with Burrows-Wheeler transform. Bioinformatics 25(14):1754-1760.

Limburg PA, Weider $\amalg$. 2002. Ancient DNA in the resting egg bank of a microcrustacean can serve as a palaeolimnological database. Proc Biol Sci. 269(1488):281-287.

Llopart A, Herrig D, Brud E, Stecklein Z. 2014. Sequential adaptive introgression of the mitochondrial genome in Drosophila yakuba and Drosophila santomea. Mol Ecol. 23(5):1124-1136.

Lomsadze A, Ter-Hovhannisyan V, Chernoff YO, Borodovsky M. 2005. Gene identification in novel eukaryotic genomes by self-training algorithm. Nucleic Acids Res. 33(20):6494-6506.

Ma X, Hu W, Smilauer P, Yin M, Wolinska J. 2019. Daphnia galeata and $D$. dentifera are geographically and ecologically separated whereas their hybrids occur in intermediate habitats: a survey of 44 Chinese lakes. Mol Ecol. 28(4):785-802.

Marçais G, Kingsford C. 2011. A fast, lock-free approach for efficient parallel counting of occurrences of k-mers. Bioinformatics 27(6):764-770.

Marková S, Dufresne F, Manca M, Kotlík P. 2013. Mitochondrial capture misleads about ecological speciation in the Daphnia pulex complex. PLoS One 8(7):e69497.

Martin SH, Davey JW, Salazar C, Jiggins CD. 2019. Recombination rate variation shapes barriers to introgression across butterfly genomes. PLoS Biol. 17(2):e2006288.

Martin SH, et al. 2013. Genome-wide evidence for speciation with gene flow in Heliconius butterflies. Genome Res. 23(11):1817-1828.

Martin SH, et al. 2020. Whole-chromosome hitchhiking driven by a malekilling endosymbiont. PLoS Biol. 18(2):e3000610.

Martins Ribeiro M, et al. 2019. Mitogenome of Daphnia laevis (Cladocera, Daphniidae) from Brazil. Mitochondrial DNA B. 4(1):194-196.

McKenna A, et al. 2010. The Genome Analysis Toolkit: a MapReduce framework for analyzing next-generation DNA sequencing data. Genome Res. 20(9):1297-1303.

Meier Jl, et al. 2017. Ancient hybridization fuels rapid cichlid fish adaptive radiations. Nat Commun. 8:1-11.

Melo-Ferreira J, et al. 2014. The elusive nature of adaptive mitochondrial DNA evolution of an arctic lineage prone to frequent introgression. Genome Biol Evol. 6(4):886-896.

Meng G, Li Y, Yang C, Liu S. 2019. MitoZ: a toolkit for animal mitochondrial genome assembly, annotation and visualization. Nucleic Acids Res. 47(11):e63.

Miner BE, De Meester L, Pfrender ME, Lampert W, Hairston NG. 2012. Linking genes to communities and ecosystems: Daphnia as an ecogenomic model. Proc Biol Sci. 279(1735):1873-1882.

Möst MH. 2013. Environmental change and its impact on hybridising Daphnia species complexes [doctoral thesis]. Zürich]: ETH.

Nadkarni MA, Martin FE, Jacques NA, Hunter N. 2002. Determination of bacterial load by real-time PCR using a broad-range (universal) probe and primers set. Microbiology 148(Pt 1):257-266.

Nguyen L-T, Schmidt HA, Von Haeseler A, Minh BQ. 2015. IQ-TREE: a fast and effective stochastic algorithm for estimating maximum-likelihood phylogenies. Mol Biol Evol. 32(1):268-274.

Orsini L, et al. 2013. The evolutionary time machine: using dormant propagules to forecast how populations can adapt to changing environments. Trends Ecol Evol. 28(5):274-282.

Patterson N, et al. 2012. Ancient admixture in human history. Genetics 192(3):1065-1093. 
Petr M, Vernot B, Kelso J. 2019. admixr $-R$ package for reproducible analyses using ADMIXTOOLS. Bioinformatics 35(17):3194-3195.

Petrusek A, Hobæk A, et al. 2008. A taxonomic reappraisal of the European Daphnia longispina complex (Crustacea, Cladocera, Anomopoda). Zool Scr. 37(5):507-519.

Petrusek A, Seda J, Machácek J, Ruthova S, Smilauer P. 2008. Daphnia hybridization along ecological gradients in pelagic environments: the potential for the presence of hybrid zones in plankton. Philos Trans $R$ Soc Lond B Biol Sci. 363(1505):2931-2941.

Petrusek A, Thielsch A, Schwenk K. 2012. Mitochondrial sequence variation suggests extensive cryptic diversity within the Western Palearctic Daphnia longispina complex. Limnol Oceanogr. 57(6):1838-1845.

Pietrzak B, Slusarczyk M. 2006. The fate of the ephippia - Daphnia dispersal in time and space. Pol J Ecol. 54:709-714.

Pinsky ML. 2021. Genomic stability through time despite decades of exploitation in cod on both sides of the Atlantic. Proc Natl Acad Sci U S A. 118(15):e2025453118.

Poplin R, et al. 2018. Scaling accurate genetic variant discovery to tens of thousands of samples. bioRxiv. doi:10.1101/201178.

Rafajlović M, Emanuelsson A, Johannesson K, Butlin RK, Mehlig B. 2016. A universal mechanism generating clusters of differentiated loci during divergence-with-migration. Evolution 70(7):1609-1621.

Ranwez V, Douzery EJ, Cambon C, Chantret N, Delsuc F. 2018. MACSE V2: toolkit for the alignment of coding sequences accounting for frameshifts and stop codons. Mol Biol Evol. 35(10):2582-2584.

R Core Team. 2017. R: a language and environment for statistical computing. Vienna (Austria): R Foundation for Statistical Computing.

Reich D, Thangaraj K, Patterson N, Price AL, Singh L. 2009. Reconstructing Indian population history. Nature 461(7263):489-494.

Rellstab C, Keller B, Girardclos S, Anselmetti FS, Spaak P. 2011. Anthropogenic eutrophication shapes the past and present taxonomic composition of hybridizing Daphnia in unproductive lakes. Limnol Oceanogr. 56(1):292-302.

Revell $\sqcup$. 2012. phytools: an R package for phylogenetic comparative biology (and other things). Methods Ecol Evol. 3(2):217-223.

Riesch R, et al. 2017. Transitions between phases of genomic differentiation during stick-insect speciation. Nat Ecol Evol. 1:82.

Runemark A, Eroukhmanoff F, Nava-Bolanos A, Hermansen JS, Meier JI. 2018. Hybridization, sex-specific genomic architecture and local adaptation. Phil Trans R Soc B. 373:20170419.

Runemark A, Trier CN, et al. 2018. Variation and constraints in hybrid genome formation. Nat Ecol Evol. 2(3):549-556.

Rusek J, et al. 2015. New possibilities arise for studies of hybridization: SNP-based markers for the multi-species Daphnia longispina complex derived from transcriptome data. J Plankton Res. 37(3):626-635.

Sarver BA, et al. 2021. Diversification, introgression, and rampant cytonuclear discordance in rocky mountains Chipmunks (Sciuridae: Tamias). Syst Biol. 70(5):908-921.

Schreiber D, Pfenninger M. 2021. Genomic divergence landscape in recurrently hybridizing Chironomus sister taxa suggests stable steady state between mutual gene flow and isolation. Evol Lett. 5(1):86-100.

Schwenk K. 1993. Interspecific hybridization in Daphnia: distinction and origin of hybrid matrilines. Mol Biol Evol. 10(6):1289-1302.

Schwenk K, Bijl M, Menken SBJ. 2001. Experimental interspecific hybridization in Daphnia. Hydrobiologia 442(1/3):67-73.

Schwenk K, et al. 1998. Genetic markers, genealogies and biogeographic patterns in the cladocera. Aquat Ecol. 32(1):37-51.

Schwenk K, Posada D, Hebert PDN. 2000. Molecular systematics of European Hyalodaphnia: the role of contemporary hybridization in ancient species. Proc Biol Sci. 267(1455):1833-1842.

Sedlazeck FJ, Rescheneder P, Von Haeseler A. 2013. NextGenMap: fast and accurate read mapping in highly polymorphic genomes. Bioinformatics 29(21):2790-2791.
Seidendorf B, Boersma M, Schwenk K. 2007. Evolutionary stoichiometry: the role of food quality for clonal differentiation and hybrid maintenance in a Daphnia species complex. Limnol Oceanogr. 52(1):385-394.

Simão FA, Waterhouse RM, loannidis P, Kriventseva EV, Zdobnov EM. 2015. BUSCO: assessing genome assembly and annotation completeness with single-copy orthologs. Bioinformatics 31(19):3210-3212.

Skage M, et al. 2007. Intra-specific rDNA-ITS restriction site variation and an improved protocol to distinguish species and hybrids in the Daphnia longispina complex. Hydrobiologia 594(1):19-32.

Slager DL, et al. 2020. Cryptic and extensive hybridization between ancient lineages of American crows. Mol Ecol. 29(5):956-969.

Sloan DB, Havird JC, Sharbrough J. 2017. The on-again, off-again relationship between mitochondrial genomes and species boundaries. Mol Ecol. 26(8):2212-2236.

Smit AFA, Hubley R. 2015. RepeatModeler Version 1.0. Available from: https://www.repeatmasker.org/RepeatModeler/. Accessed November 2021.

Smit AFA, Hubley R, Green P. 2013-2015. RepeatMasker Open-4.0. Available from: https://www.repeatmasker.org/RepeatMasker/. Accessed November 2021.

Spaak P. 2004. Spatial and temporal patterns of sexual reproduction in a hybrid Daphnia species complex. Spatial and temporal patterns of sexual reproduction in a hybrid Daphnia species complex. J Plankton Res. 26(6):625-635.

Spaak P, Fox J, Hairston NG. 2012. Modes and mechanisms of a Daphnia invasion. Proc Biol Sci. 279(1740):2936-2944.

Spaak P, Hoekstra JR. 1997. Fish predation on a Daphnia hybrid species complex: a factor explaining species coexistence? Limnol Oceanogr. 42(4):753-762.

Stanke M, Diekhans M, Baertsch R, Haussler D. 2008. Using native and syntenically mapped cDNA alignments to improve de novo gene finding. Bioinformatics 24(5):637-644.

Stephens JD, Rogers WL, Mason CM, Donovan LA, Malmberg RL. 2015. Species tree estimation of diploid Helianthus (Asteraceae) using target enrichment. Am J Bot. 102(6):910-920.

Taylor DJ, Hebert PDN, Colbourne JK. 1996. Phylogenetics and evolution of the Daphnia longispina group (Crustacea) based on 12S rDNA sequence and allozyme variation. Mol Phylogenet Evol. 5(3):495-510.

Thielsch A, Brede N, Petrusek A, De Meester L, Schwenk K. 2009. Contribution of cyclic parthenogenesis and colonization history to population structure in Daphnia. Mol Ecol. 18(8):1616-1628.

Thielsch A, Knell A, Mohammadyari A, Petrusek A, Schwenk K. 2017. Divergent clades or cryptic species? Mito-nuclear discordance in a Daphnia species complex. BMC Evol Biol. 17(1):227.

Thielsch A, Volker E, Kraus RHS, Schwenk K. 2012. Discrimination of hybrid classes using cross-species amplification of microsatellite loci: methodological challenges and solutions in Daphnia. Mol Ecol Resour. 12(4):697-705.

Tollrian R, Harvell CD. 1999. The ecology and evolution of inducible defenses. Princeton (NJ): Princeton University Press.

Trentini M. 1980. Chromosome numbers of nine species of Daphniidae (Crustacea, Cladocera). Genetica 54(2):221-223.

UniProt Consortium. 2019. UniProt: a worldwide hub of protein knowledge. Nucleic Acids Res. 47(D1):D506-D515.

Vaidya G, Lohman DJ, Meier R. 2011. SequenceMatrix: concatenation software for the fast assembly of multi-gene datasets with character set and codon information. Cladistics 27(2):171-180.

Van der Auwera GA, et al. 2013. From FastQ data to high-confidence variant calls: the genome analysis toolkit best practices pipeline. Curr Protoc Bioinformatics. 43:11-10.

Vergilino R, Markova S, Ventura M, Manca M, Dufresne F. 2011. Reticulate evolution of the Daphnia pulex complex as revealed by nuclear markers. Mol Ecol. 20(6):1191-1207. 
Waldvogel AM, et al. 2018. The genomic footprint of climate adaptation in Chironomus riparius. Mol Ecol. 27(6):1439-1456.

Walker BJ, et al. 2014. Pilon: an integrated tool for comprehensive microbial variant detection and genome assembly improvement. PLoS One 9(11):e112963.

Wingett SW, Andrews S. 2018. FastQ Screen: a tool for multi-genome mapping and quality control. F1000Res. 7:1338.

Wolf HG. 1987. Interspecific hybridization between Daphnia hyalina, $D$. galeata, and $D$. cucullata and seasonal abundances of these species and their hybrids. In: Forró L, Frey DG, editors. Cladocera. Dordrecht: Springer. p. 213-217.

Wolf HG, Mort MA. 1986. Interspecific hybridization underlies phenotypic variability in Daphnia populations. Oecologia 68(4):507-511.

Ye ZQ, et al. 2017. A new reference genome assembly for the microcrustacean Daphnia pulex. G3 (Bethesda) 7(5):1405-1416.
Yeaman S, Whitlock MC. 2011. The genetic architecture of adaptation under migration-selection balance. Evolution 65(7):1897-1911.

Yin MB, Giessler S, Griebel J, Wolinska J. 2014. Hybridizing Daphnia communities from ten neighbouring lakes: spatio-temporal dynamics, local processes, gene flow and invasiveness. BMC Evol Biol. $14: 80$.

Zehnder A, Gorham PR. 1960. Factors influencing the growth of Microcystis aeruginosa Kütz. Can J Microbiol. 6:645660.

Zheng X, et al. 2012. A high-performance computing toolset for relatedness and principal component analysis of SNP data. Bioinformatics 28(24):3326-3328.

Associate editor: Emmanuelle Lerat 